\title{
Late Quaternary deep-sea ostracod taxonomy of the eastern North Atlantic Ocean
}

\author{
MORIAKI YASUHARA* \& HISAYO OKAHASHI \\ School of Biological Sciences, Swire Institute of Marine Science, and Department of Earth Sciences, \\ University of Hong Kong, Kadoorie Biological Sciences Building, Pokfulam Road, Hong Kong SAR, China \\ *Corresponding author (e-mail: yasuhara@hku.hk)
}

\begin{abstract}
Taxonomic revision and re-evaluation of the eastern North Atlantic deep-sea ostracods are conducted based on late Quaternary sediments from Ocean Drilling Program (ODP) Hole 982A, Rockall Plateau, eastern North Atlantic. Twenty-one genera and 51 species were examined and (re-)illustrated with highresolution scanning electron microscopy images. Six new species are described: Polycope lunaris, Argilloecia labri, Bythoceratina nuda, Cytheropteron colesoabyssorum, Cytheropteron colesopunctatum and Cytheropteron paramediotumidum. Excellent fossil ostracod preservation in this sediment core enabled us to provide a robust taxonomic baseline of the eastern North Atlantic deep-sea ostracods for application to palaeoceanographical, palaeoecological and biogeographical studies.
\end{abstract}

KEYWORDS: deep-sea, Ostracoda, taxonomy, Quaternary, eastern North Atlantic, upper bathyal

\section{INTRODUCTION}

North Atlantic deep-sea ostracods have been well investigated (e.g. Brady, 1880; Whatley \& Coles, 1987; Coles \& Whatley, 1989; Dingle \& Lord, 1990; Cronin \& Raymo, 1997; Cronin et al., 1999; Didié et al., 2002; Yasuhara \& Cronin, 2008; Yasuhara et al., 2008, 2009a; Alvarez Zarikian, 2009; Yamaguchi \& Norris, 2012). However, detailed taxonomic studies using the scanning electron microscope (SEM) are still limited and concentrated on the ostracod faunas from the lower bathyal and abyssal zones (i.e. $>2000 \mathrm{~m}$ water depth) (Whatley \& Coles, 1987; Coles \& Whatley, 1989; Coles et al., 1994; Alvarez Zarikian, 2009). Thus, little is known on the bathyal North Atlantic ostracod taxonomy, compared to well-investigated Mediterranean bathyal fauna (Bonaduce et al., 1976; Colalongo \& Pasini, 1980; Aiello et al., 2000; Guernet, 2005; Aiello \& Barra, 2010), even though bathyal faunas are usually much more diverse than abyssal faunas (e.g. Yasuhara et al., 2012). Furthermore, there is some taxonomic confusion in North Atlantic ostracod taxonomic, faunal and palaeoceanographic studies in which a same species has often been called by several different names (see synonymy lists in the present study).

Recently, Yasuhara et al. (2009b) conducted a comprehensive taxonomic revision of western North Atlantic Quaternary deep-sea ostracods using a sediment core taken from the upper bathyal zone with high-resolution SEM images of 87 species and a detailed literature survey. However, a comparable in-depth taxonomic study has not been undertaken previously for the upper bathyal zone of the eastern North Atlantic.

Ocean Drilling Program (ODP) Hole 982A gave us an ideal opportunity to study eastern North Atlantic deep-sea ostracods from the upper bathyal zone in detail, because its sediments have an abundant, diverse and well-preserved ostracod fauna. Here we investigate late Quaternary ODP 982A ostracod taxonomy using high-resolution SEM images to reduce taxonomic confusion of North Atlantic bathyal ostracods. In addition, we briefly discuss similarity of bathyal ostracod faunas among the western and eastern North Atlantic, the Mediterranean, and the western North Pacific.

\section{MATERIALS AND METHODS}

A total of 47 samples of ODP Hole $982 \mathrm{~A} \quad\left(57^{\circ} 30.992^{\prime} \mathrm{N}\right.$, $15^{\circ} 52.001^{\prime} \mathrm{W}, 1135.3 \mathrm{~m}$ water depth; Rockall Plateau, eastern North Atlantic) covering the past 230000 years and Marine Isotope Stages (MIS) 1-7 (Venz et al., 1999) were examined for ostracod taxonomy. The full information for the samples and specimens used for the present study is shown in Tables 1 and 2 . Uncoated specimens were digitally imaged with a Philips XL-30 environmental SEM. High-resolution figures of ostracod SEM images (Figs 2-16) are available at Dryad (http://datadryad.org/; http://doi.org/10.5061/dryad.sc193. We follow the higher classification scheme of the World Register of Marine Species (WoRMS: http://www.marinespecies.org/) with certain modifications.

Repository. Figured specimens are deposited in the National Museum of Natural History (Washington DC, catalogue numbers USNM 603625-USNM 603760).

Abbreviations. LV, left valve; RV, right valve; L, length (mm); $\mathrm{H}$, height ( $\mathrm{mm})$.

\section{SYSTEMATIC PALAEONTOLOGY}

Class Ostracoda Latreille, 1802

Subclass Myodocopa Sars, 1866

Order Halocyprida Dana, 1853

Suborder Cladocopina Sars, 1866

Superfamily Polycopidea Sars, 1866

Family Polycopidae Sars, 1866

Genus Polycope Sars, 1866

Type species. Polycope orbicularis Sars, 1866

Remarks. We use the genus name Polycope in a broad sense following typical deep-sea ostracod taxonomy, but note that recent zoological studies, for example Karanovic \& Brandão (2012), divide the genus into several separate genera based on soft parts, which are not preserved in fossil ostracods.

\section{Polycope martinezi (Karanovic \& Brandão, 2012)}

(Fig. 2A)

2001 Polycope sp. cf. P. arcys Joy \& Clark; Didié \& Bauch: 104, pl. 1, fig. 28 (as erratum for Didié \& Bauch, 2000). 
Table 1. Detailed information of the specimens used for the present study.

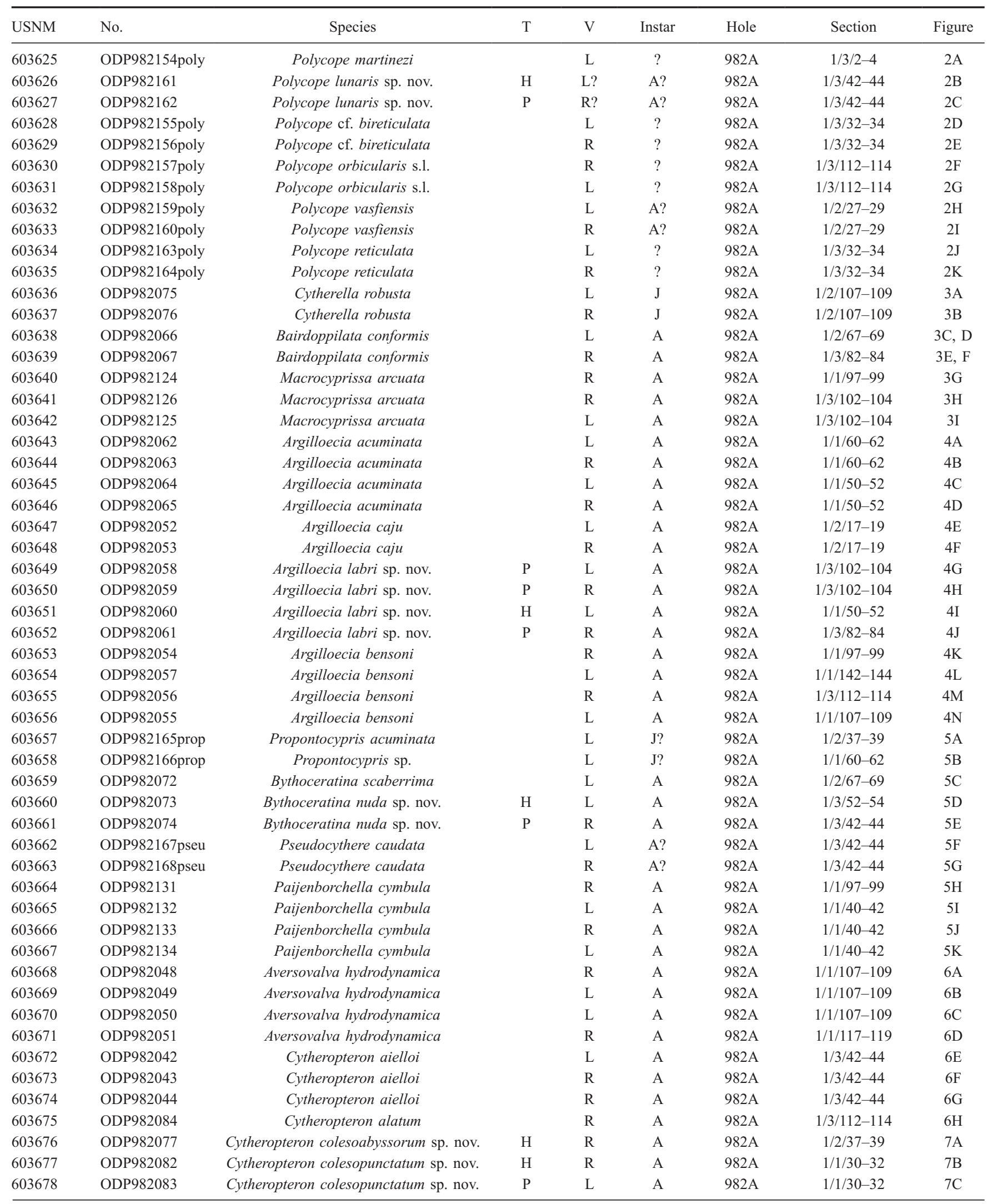


Table 1. (Continued)

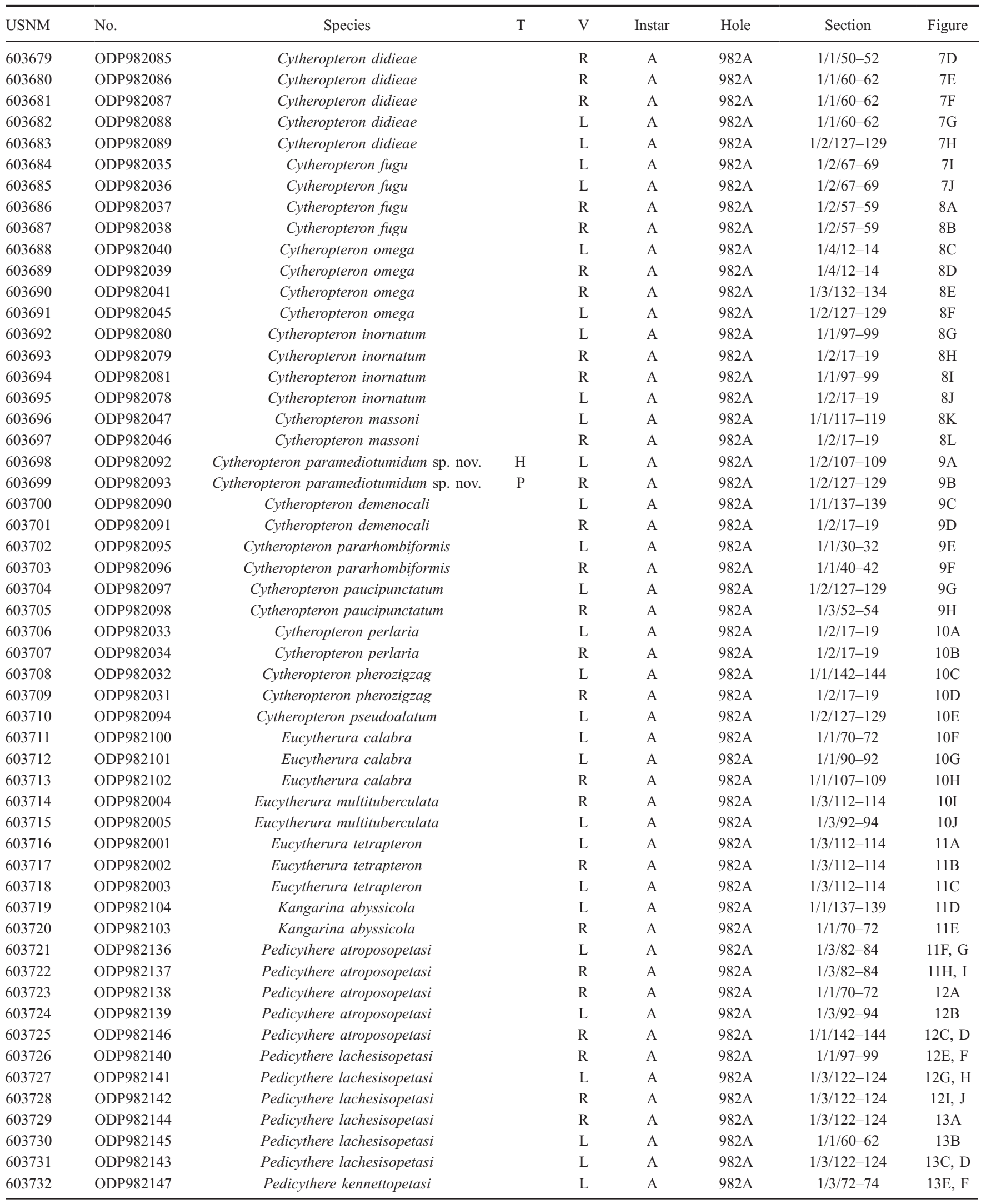


Table 1. (Continued)

\begin{tabular}{|c|c|c|c|c|c|c|c|c|}
\hline USNM & No. & Species & $\mathrm{T}$ & $\mathrm{V}$ & Instar & Hole & Section & Figure \\
\hline 603734 & ODP982149pedi & Pedicythere klothopetasi & & $\mathrm{R}$ & A & $982 \mathrm{~A}$ & $1 / 1 / 80-82$ & $13 \mathrm{H}$ \\
\hline 603736 & ODP982151 & Pedicythere klothopetasi & & $\mathrm{R}$ & $\mathrm{A}$ & $982 \mathrm{~A}$ & $1 / 1 / 80-82$ & $14 \mathrm{~A}$ \\
\hline 603737 & ODP982152 & Pedicythere klothopetasi & & $\mathrm{L}$ & A & $982 \mathrm{~A}$ & $1 / 1 / 70-72$ & $14 \mathrm{~B}, \mathrm{C}$ \\
\hline 603738 & ODP982153 & Pedicythere klothopetasi & & $\mathrm{R}$ & A & $982 \mathrm{~A}$ & $1 / 1 / 70-72$ & $14 \mathrm{D}, \mathrm{E}$ \\
\hline 603740 & ODP982127-2 & Cluthia sp. & & $\mathrm{L}$ & A & $982 \mathrm{~A}$ & $1 / 3 / 12-14$ & $14 \mathrm{G}$ \\
\hline 603741 & ODP982128 & Cluthia sp. & & $\mathrm{R}$ & $\mathrm{A}$ & $982 \mathrm{~A}$ & $1 / 3 / 92-94$ & $14 \mathrm{H}$ \\
\hline 603742 & ODP982129-1 & Cluthia sp. & & $\mathrm{L}$ & $\mathrm{A}$ & $982 \mathrm{~A}$ & $1 / 3 / 92-94$ & $14 \mathrm{I}$ \\
\hline 603743 & ODP982130 & Cluthia sp. & & $\mathrm{R}$ & $\mathrm{A}$ & $982 \mathrm{~A}$ & $1 / 3 / 92-94$ & $14 \mathrm{~J}$ \\
\hline 603744 & ODP982123 & Loxoconchidea minima & & $\mathrm{L}$ & A & $982 \mathrm{~A}$ & $1 / 3 / 12-14$ & $15 \mathrm{~A}$ \\
\hline 603745 & ODP982135 & Paracytherois bondi & & $\mathrm{R}$ & A & $982 \mathrm{~A}$ & $1 / 1 / 80-82$ & $15 \mathrm{~B}, \mathrm{C}$ \\
\hline 603750 & ODP982173rock & Arcacythere enigmatica & & $\mathrm{R}$ & A & $982 \mathrm{~A}$ & $1 / 2 / 67-69$ & $15 \mathrm{H}$ \\
\hline 603751 & ODP982174rock & Arcacythere enigmatica & & $\mathrm{L}$ & $\mathrm{A}$ & $982 \mathrm{~A}$ & $1 / 3 / 92-94$ & $15 \mathrm{I}$ \\
\hline 603752 & ODP982027 & Echinocythereis echinata & & $\mathrm{R}$ & $\mathrm{A}$ & $982 \mathrm{~A}$ & $1 / 1 / 142-144$ & $16 \mathrm{~A}$ \\
\hline 603753 & ODP982028 & Echinocythereis echinata & & $\mathrm{R}$ & A & $982 \mathrm{~A}$ & $1 / 1 / 142-144$ & $16 \mathrm{~B}$ \\
\hline 603754 & ODP982029 & Echinocythereis echinata & & $\mathrm{L}$ & A & $982 \mathrm{~A}$ & $1 / 3 / 72-74$ & $16 \mathrm{C}$ \\
\hline 603755 & ODP982030 & Echinocythereis echinata & & $\mathrm{L}$ & A & $982 \mathrm{~A}$ & $1 / 3 / 72-74$ & $16 \mathrm{D}$ \\
\hline 603756 & ODP982022 & Henryhowella asperrima & & $\mathrm{L}$ & $\mathrm{A}$ & $982 \mathrm{~A}$ & $1 / 1 / 137-139$ & $16 \mathrm{E}$ \\
\hline 603757 & ODP982023 & Henryhowella asperrima & & $\mathrm{R}$ & $\mathrm{A}$ & $982 \mathrm{~A}$ & $1 / 1 / 137-139$ & $16 \mathrm{~F}$ \\
\hline 603758 & ODP982026 & Henryhowella asperrima & & $\mathrm{L}$ & $\mathrm{A}$ & $982 \mathrm{~A}$ & $1 / 1 / 0-2$ & $16 \mathrm{G}$ \\
\hline 603759 & ODP982024 & Henryhowella asperrima & & $\mathrm{R}$ & A & $982 \mathrm{~A}$ & $1 / 1 / 142-144$ & $16 \mathrm{H}, \mathrm{I}$ \\
\hline 603760 & ODP982025 & Henryhowella asperrima & & $\mathrm{L}$ & $\mathrm{A}$ & $982 \mathrm{~A}$ & 1/1/107-109 & $16 \mathrm{~J}, \mathrm{~K}$ \\
\hline
\end{tabular}

All specimens from late Quaternary sediments. Core samples are specified by standard ODP notation (core/section/interval). USNM, catalog number; No., M.Y.'s personal catalog number. T, type (P, paratype; H, holotype); V, valve (L, left; R, right); A, adult; J, juvenile.

2009 b Polycope arcys Joy \& Clark; Yasuhara et al.: 881, pl. 1, fig. 6. 2012 Archypolycope martinezi Karanovic \& Brandão: 348, figs 20-24.

Remarks. Polycope martinezi is very similar to the Arctic species Polycope arcys, but distinguished by lacking obvious lateral spines and having finer reticulation. P. martinezi was originally reported from the equatorial Atlantic (Karanovic \& Brandão, 2012) and is also known from the North Atlantic (Didié \& Bauch, 2000, 2001; Yasuhara et al. 2009b).

\section{Polycope lunaris sp. nov.}

(Fig. 2B-C)

2001 Polycope sp. Didié \& Bauch: 103, pl. 1, fig. 27 (as erratum for Didié \& Bauch, 2000).

Derivation of name. From Latin lunaris (adjective; nominative singular; gender, neutral) $=$ lunar.

Diagnosis. A small, heavily calcified Polycope species with welldeveloped primary reticulation.

Holotype. LV, USNM 603626 (ODP982161) (Fig. 2B).

Paratype. RV, USNM 603627 (ODP982162).
Type locality and horizon. ODP 982A, 1/3/42-44.

Description. Carapace heavily calcified, small in size. Outline rounded in lateral view. Lateral surface ornamented with welldeveloped, rounded primary reticulation. Anteroventral ridge thick, well developed and bearing no reticulation. Internal features as for genus.

Dimensions. USNM 603626 (ODP982161) (Holotype), $\mathrm{L}=0.378$, $\mathrm{H}=0.343$; USNM 603627 (ODP982162) (Paratype), $\mathrm{L}=0.353$, $\mathrm{H}=0.328$.

Remarks. This species is distinctive from any other Polycope species by having a heavily calcified carapace with well-developed, rounded primary reticulation and thick anteroventral ridge.

\section{Polycope cf. bireticulata Joy \& Clark, 1977}

(Fig. 2D-E)

2009 b Polycope cf. bireticulata Joy \& Clark; Yasuhara et al.: 881, pl. 1, figs 3 and 4.

Remarks. ODP 982A specimens have better developed reticulation compared to the specimens from the western North Atlantic (Yasuhara et al., 2009b), considered here to be 
Table 2. The list of ODP 982A samples used for the present study.

\begin{tabular}{|c|c|c|c|c|c|c|c|}
\hline Core & Section & $\begin{array}{l}\text { Interval } \\
\text { (top: } \mathrm{cm} \text { ) }\end{array}$ & $\begin{array}{c}\text { Interval } \\
\text { (bottom: } \mathrm{cm} \text { ) }\end{array}$ & $\begin{array}{l}\mathrm{MCD} \\
(\mathrm{cm})\end{array}$ & $\begin{array}{c}\text { Age } \\
(\text { ka BP) }\end{array}$ & $\mathrm{N}$ & S \\
\hline 1 & 1 & 0 & 2 & 0 & 0.0 & 87 & 10 \\
\hline 1 & 1 & 10 & 12 & 10 & 1.0 & 119 & 15 \\
\hline 1 & 1 & 20 & 22 & 20 & 2.2 & 107 & 14 \\
\hline 1 & 1 & 30 & 32 & 30 & 3.4 & 204 & 19 \\
\hline 1 & 1 & 40 & 42 & 40 & 5.2 & 276 & 26 \\
\hline 1 & 1 & 50 & 52 & 50 & 7.2 & 221 & 35 \\
\hline 1 & 1 & 60 & 62 & 60 & 10.0 & 231 & 40 \\
\hline 1 & 1 & 70 & 72 & 70 & 14.9 & 184 & 39 \\
\hline 1 & 1 & 80 & 82 & 80 & 19.9 & 141 & 25 \\
\hline 1 & 1 & 90 & 92 & 90 & 24.9 & 140 & 31 \\
\hline 1 & 1 & 97 & 99 & 97 & 28.3 & 237 & 42 \\
\hline 1 & 1 & 107 & 109 & 107 & 33.3 & 234 & 30 \\
\hline 1 & 1 & 117 & 119 & 117 & 38.3 & 241 & 25 \\
\hline 1 & 1 & 127 & 129 & 127 & 43.2 & 312 & 29 \\
\hline 1 & 1 & 137 & 139 & 137 & 48.2 & 259 & 28 \\
\hline 1 & 1 & 142 & 144 & 142 & 50.7 & 354 & 34 \\
\hline 1 & 2 & 7 & 9 & 157 & 58.1 & 264 & 40 \\
\hline 1 & 2 & 17 & 19 & 167 & 63.1 & 283 & 34 \\
\hline 1 & 2 & 27 & 29 & 177 & 68.0 & 268 & 37 \\
\hline 1 & 2 & 37 & 39 & 187 & 73.0 & 228 & 34 \\
\hline 1 & 2 & 47 & 49 & 197 & 77.9 & 227 & 35 \\
\hline 1 & 2 & 57 & 59 & 207 & 82.9 & 214 & 28 \\
\hline 1 & 2 & 67 & 69 & 217 & 87.9 & 195 & 32 \\
\hline 1 & 2 & 77 & 79 & 227 & 92.8 & 155 & 32 \\
\hline 1 & 2 & 87 & 89 & 237 & 97.8 & 264 & 29 \\
\hline 1 & 2 & 97 & 99 & 247 & 102.8 & 123 & 25 \\
\hline 1 & 2 & 107 & 109 & 257 & 107.7 & 320 & 25 \\
\hline 1 & 2 & 117 & 119 & 267 & 112.7 & 201 & 21 \\
\hline 1 & 2 & 127 & 129 & 277 & 117.6 & 237 & 28 \\
\hline 1 & 2 & 137 & 139 & 287 & 122.6 & 148 & 24 \\
\hline 1 & 3 & 2 & 4 & 302 & 130.0 & 220 & 30 \\
\hline 1 & 3 & 12 & 14 & 312 & 136.3 & 169 & 25 \\
\hline 1 & 3 & 22 & 24 & 322 & 146.5 & 161 & 27 \\
\hline 1 & 3 & 32 & 34 & 332 & 156.8 & 139 & 28 \\
\hline 1 & 3 & 42 & 44 & 342 & 165.0 & 195 & 27 \\
\hline 1 & 3 & 52 & 54 & 352 & 172.5 & 152 & 28 \\
\hline 1 & 3 & 62 & 64 & 362 & 179.6 & 228 & 26 \\
\hline 1 & 3 & 72 & 74 & 372 & 184.9 & 128 & 25 \\
\hline 1 & 3 & 82 & 84 & 382 & 190.2 & 229 & 28 \\
\hline 1 & 3 & 92 & 94 & 392 & 195.3 & 164 & 31 \\
\hline 1 & 3 & 102 & 104 & 402 & 200.4 & 294 & 43 \\
\hline 1 & 3 & 112 & 114 & 412 & 205.6 & 198 & 34 \\
\hline 1 & 3 & 122 & 124 & 422 & 210.7 & 367 & 39 \\
\hline 1 & 3 & 132 & 134 & 432 & 215.8 & 131 & 27 \\
\hline 1 & 3 & 142 & 144 & 442 & 220.9 & 164 & 27 \\
\hline 1 & 4 & 2 & 4 & 452 & 226.0 & 202 & 29 \\
\hline 1 & 4 & 12 & 14 & 462 & 231.1 & 161 & 29 \\
\hline
\end{tabular}

$\mathrm{N}$, number of ostracode specimens per sample; S, number of ostracode species per sample. Chronology from Venz et al. (1999)

intraspecific variation. This species is similar to $P$. bireticulata, but the latter has a more evenly rounded outline and different alignment of muri (Yasuhara et al. in press $b$ ).

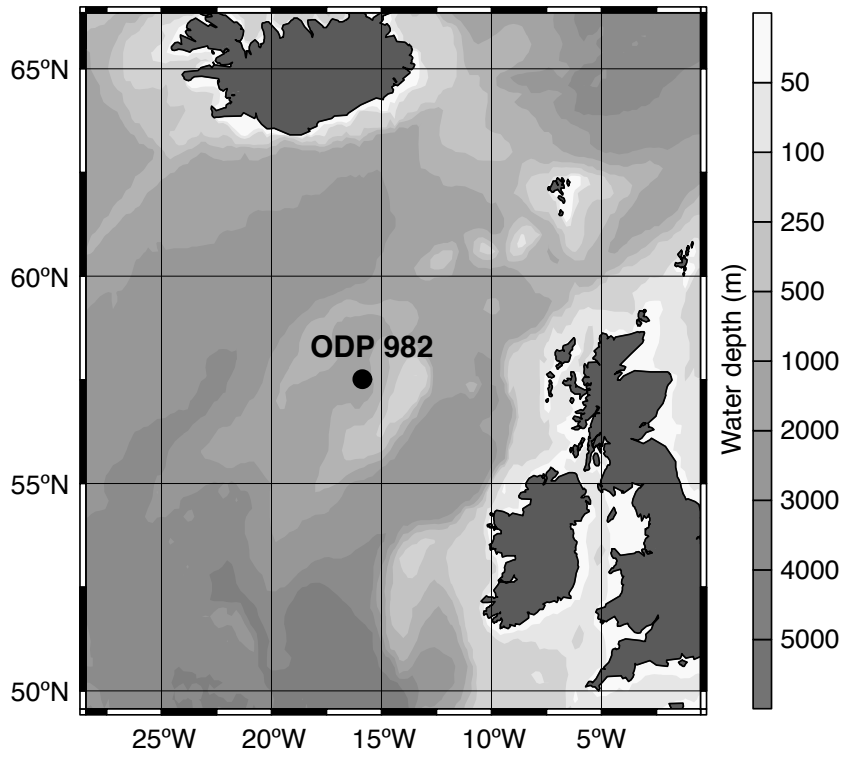

Fig. 1. Locality map of ODP Hole 982A.

Polycope orbicularis s.1. Sars, 1866

(Fig. 2F-G)

2009 b Polycope cf. orbicularis Sars; Yasuhara et al.: 881, pl. 1, fig. 5. 2009 b Polycope orbicularis s.1. Sars; Yasuhara et al.: 881.

2009 Polycope orbicularis Sars; Alvarez Zarikian: 3, pl. P1, fig. 7.

Remarks. Polycope orbicularis s.1. is discussed in Yasuhara et al. $(2009 b)$.

Polycope vasfiensis Sissingh, 1972

(Fig. 2H-I)

1972 Polycope vasfiensis Sissingh: 68, pl. 1, fig. 6.

1976 Polycope vasfiensis Sissingh; Bonaduce et al:: 18, pl. 1, figs 6-8, text-fig. 6.

2000 Polycope vasfiensis Sissingh; Aiello et al.: 85, pl. 1, fig. 1. 2009 b Polycope vasfiensis Sissingh; Yasuhara et al.: 882, pl. 1, figs $1-2$.

\section{Polycope reticulata Müller, 1894}

(Fig. 2J-K)

1894 Polycope reticulata Müller: 235, pl. 7, figs 44, 49-50; pl. 8, fig. 20.

non 1976 Polycope reticulata Müller; Bonaduce et al.: 14, pl. 2, figs 9 and 10.

2013 Polycope reticulata Müller; Cabral \& Loureiro: 137, pl. 1, fig. 1.

Remarks. We consider that Polycope reticulata sensu Bonaduce et al. (1976) is not conspecific with $P$. reticulata Müller, 1894 because the original sketch by Müller (1894, pl. 8, fig. 20) lacks secondary reticulation and has a different primary reticulation pattern and more inflated carapace.

Subclass Podocopa Müller, 1894

Order Platycopida Sars, 1866

Suborder Platycopina Sars, 1866

Superfamily Cytherelloidea Sars, 1866 


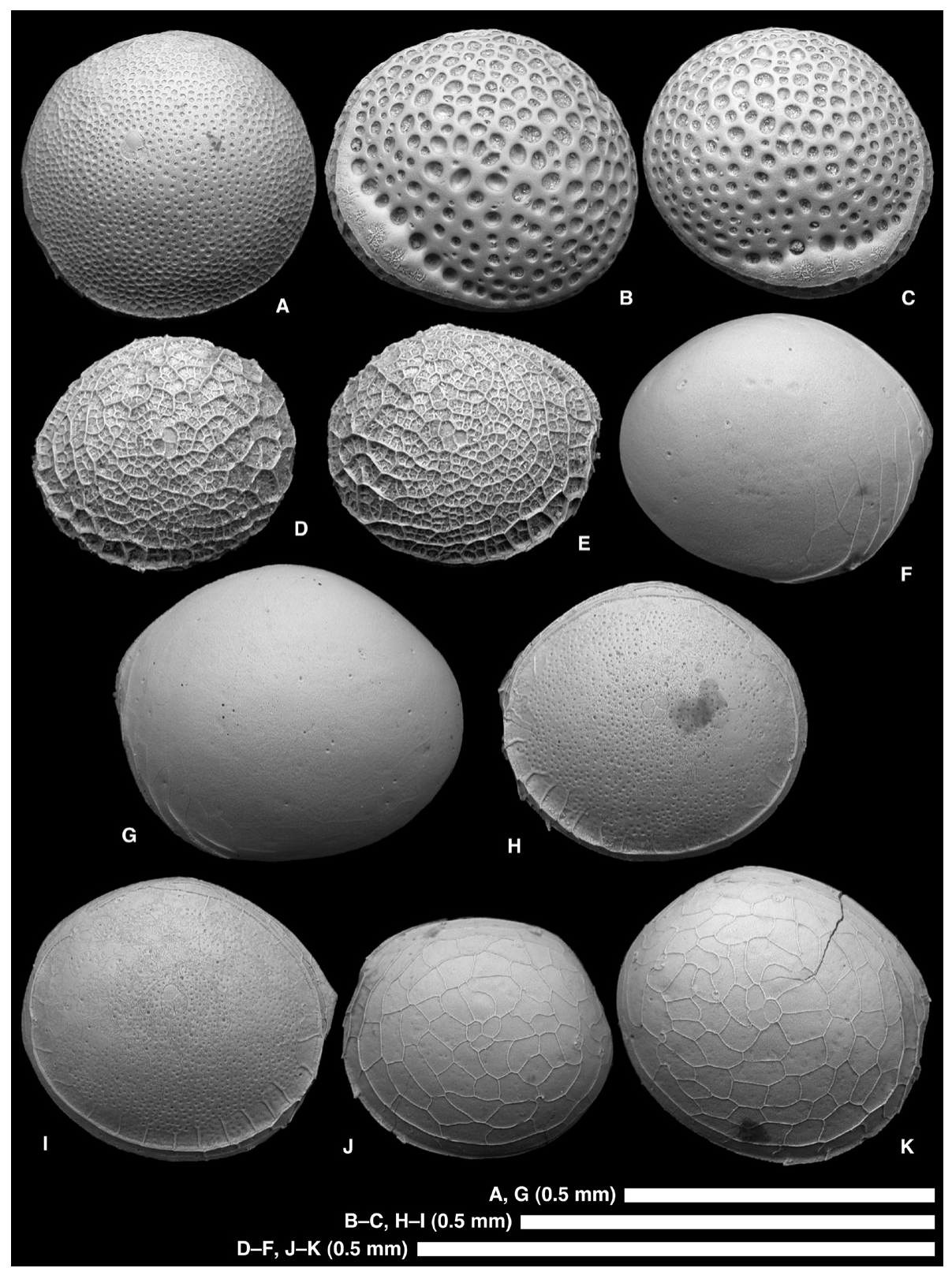

Fig. 2. Scanning electron microscope (SEM) images of Polycope species. A, Polycope martinezi (Karanovic \& Brandão, 2012), USNM 603625 (ODP982154poly); LV from 1/3/2-4. B-C, Polycope lunaris sp. nov.: B, Holotype USNM 603626 (ODP982161); adult? LV? from 1/3/42-44; C, Paratype USNM 603627 (ODP982162); adult? RV? from 1/3/42-44. D-E, Polycope cf. bireticulata Joy \& Clark, 1977: D, USNM 603628 (ODP982155poly); LV from 1/3/32-34; E, USNM 603629 (ODP982156poly); RV from 1/3/32-34. F-G, Polycope orbicularis s.1. Sars, 1866: F, USNM 603630 (ODP982157poly); RV from 1/3/112-114; G, USNM 603631 (ODP982158poly); LV from 1/3/112-114. H-I, Polycope vasfiensis Sissingh, 1972: H, USNM 603632 (ODP982159poly); adult? LV from 1/2/27-29; I, USNM 603633 (ODP982160poly); adult? RV from 1/2/27-29. J-K, Polycope reticulata Müller, 1894: J, USNM 603634 (ODP982163poly); LV from 1/3/32-34; K, USNM 603635 (ODP982164poly); RV from 1/3/32-34. All lateral views. All specimens from late Quaternary section of ODP Hole 982A, Rockall Plateau, eastern North Atlantic. Scale bars represent $0.5 \mathrm{~mm}$.

Family Cytherellidae Sars, 1866

Genus Cytherella Jones, 1849

Type species. Cytherina ovata Roemer, 1841

(designated by Ulrich, 1894)

Cytherella robusta Colalongo \& Pasini, 1980

(Fig. 3A-B)

1979 Cytherella sp. 11 Ducasse \& Peypouquet: pl. 1, figs 3-4.
1980 Cytherella robusta Colalongo \& Pasini: 78, pl. 6, figs 4-10.

1996 Cytherella robusta Colalongo \& Pasini; Aiello et al.: 184, pl. 2, figs 4-5, 8-12.

2001 Cytherella serratula (Brady); Didié \& Bauch: 104, pl. 1, fig. 5 (erratum for Didié \& Bauch, 2000).

2001 Cytherella sp. 1 Didié \& Bauch: 104, pl. 1, fig. 6 (erratum for Didié \& Bauch, 2000). 


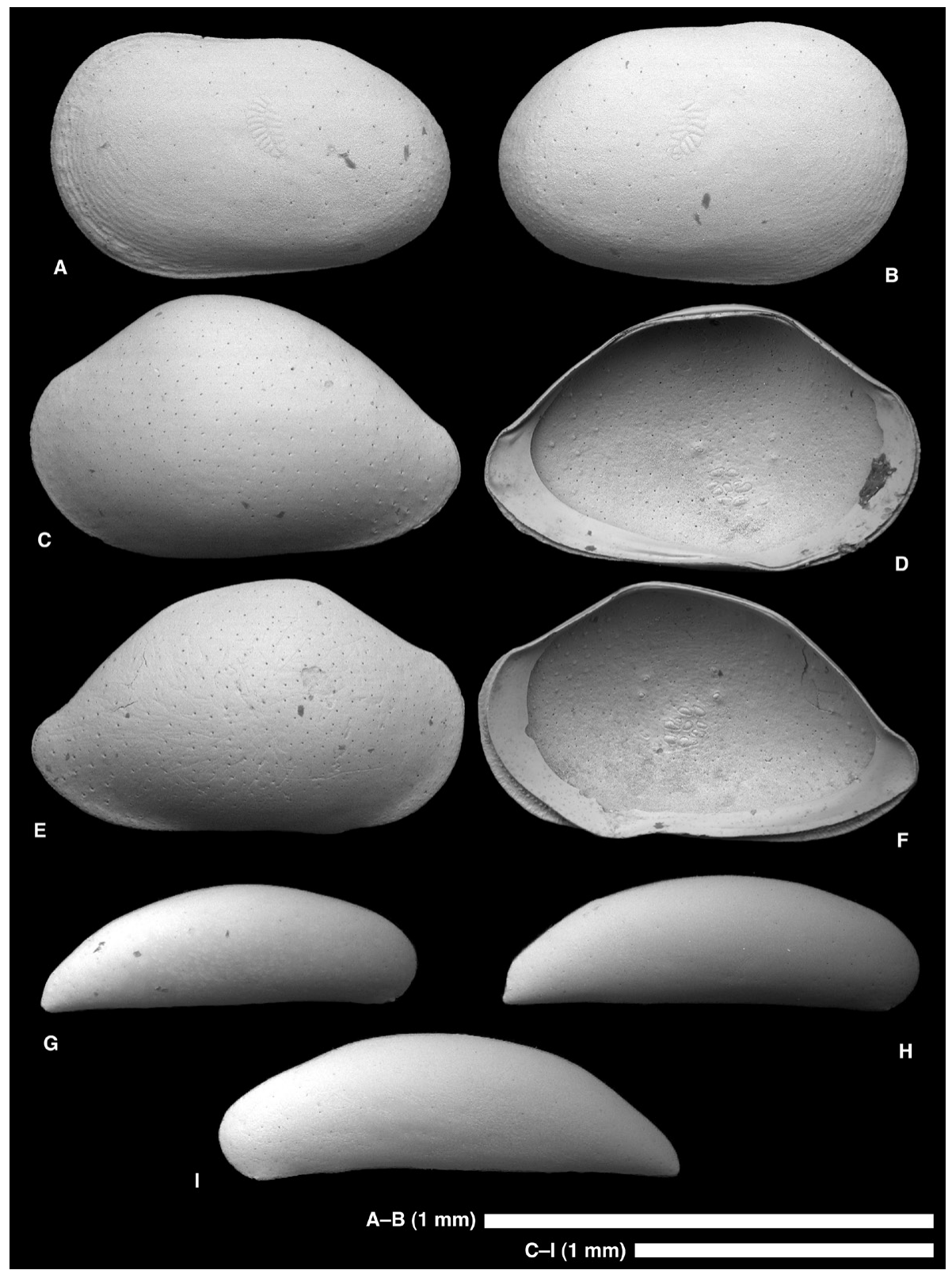

Fig. 3. SEM images of Cytherella robusta Colalongo \& Pasini, 1980, Bairdoppilata conformis (Terquem, 1878) and Macrocyprissa arcuata (Colalongo \& Pasini, 1980). A-B, Cytherella robusta Colalongo \& Pasini, 1980: A, USNM 603636 (ODP982075); juvenile LV from 1/2/107-109; B, USNM 603637 (ODP982076); juvenile RV from 1/2/107-109. C-F, Bairdoppilata conformis (Terquem, 1878): C-D, USNM 603638 (ODP982066); adult LV from 1/2/67-69; E-F, USNM 603639 (ODP982067); adult RV from 1/3/82-84. G-I, Macrocyprissa arcuata (Colalongo \& Pasini, 1980): G, USNM 603640 (ODP982124); adult RV from 1/1/97-99; H, USNM 603641 (ODP982126); adult RV from 1/3/102-104; I, USNM 603642 (ODP982125); adult LV from 1/3/102-104. A-C, E, G-I, lateral views; D, F, internal views. All specimens from late Quaternary section of ODP Hole 982A, Rockall Plateau, eastern North Atlantic. Scale bars represent $1 \mathrm{~mm}$.

2001 Cytherella sp. 2 Didié \& Bauch: 104, pl. 1, fig. 7 (erratum for Didié \& Bauch, 2000).

2009 Cytherella sp. Alvarez Zarikian: 7, pl. P10, fig. 5.

2009 b Cytherella robusta s.l. Colalongo \& Pasini; Yasuhara et al.: 882, pl. 1, figs 7-12.

2013 Cytherella robusta Colalongo \& Pasini; Cabral \& Loureiro: 137, pl. 1, fig. 3 .

Remarks. Juvenile specimens of Cytherella robusta are shown here. As discussed in Yasuhara \& Okahashi (in press), this species has certain intraspecific variation. A comprehensive synonymy list in Aiello et al. (1996b), Yasuhara et al. (2009b) and Yasuhara \& Okahashi (in press).

Order Podocopida Sars, 1866

Suborder Bairdiocopina Gründel, 1967

Superfamily Bairdioidea Sars, 1866

Family Bairdiidae Sars, 1866

Remarks. We follow Maddocks' (1969) genus-level taxonomy for this family. 
Genus Bairdoppilata Coryell, Sample \& Jennings (1935)

Type species. Bairdoppilata martyni Coryell, Sample \& Jennings (1935)

\section{Bairdoppilata conformis (Terquem, 1878)}

(Fig. 3C-F)

1878 Bairdia subdeltoidea var. conformis Terquem: 93, pl. 10, fig. $17 \mathrm{a}-\mathrm{c}$.

1962 Bairdia conformis Terquem; Ruggieri: 13.

1976 Bairdia conformis Terquem; Bonaduce et al:: 22, pl. 6, figs $5-10$.

2000 Bairdoppilata conformis (Terquem); Aiello et al.: 85, pl. 1, fig. 2.

2003 Bairdoppilata conformis (Terquem); Sciuto: 182, fig. 2a.

2008 Bairdoppilata conformis (Terquem); Faranda et al.: 300, tab. 2.

2010 Bairdoppilata conformis (Terquem); Aiello \& Barra: 406.

Remarks. A comprehensive synonymy list is in Aiello et al. (2000) and supplemented herein. Our specimens are identical to that shown in Aiello et al. (2000). Our specimens are also very similar to the specimens in Terquem (1878) and Bonaduce et al. (1976), but the latter specimens have a more heavily calcified carapace and slightly more upturned caudal process. Other authors have also reported this species, but with a slightly different outline (Sciuto, 2003) or without images (Faranda et al., 2008; Aiello \& Barra, 2010). We consider all of these differences as intraspecific variation and include them in Bairdoppilata conformis. Slight differences in outlines may be due to intraspecific variation in calcification. No SEM or microscopic image of type specimens has been published.

Suborder Cypridocopina Jones, 1901

Superfamily Macrocypridoidea Müller, 1912

Family Macrocyprididae Müller, 1912

Genus Macrocyprissa Triebel, 1960

Type species. Bairdia cylindracea Bornemann, 1855

Macrocyprissa arcuata (Colalongo \& Pasini, 1980)

(Fig. 3G-I)

1980 Paramacrocypris arcuata Colalongo \& Pasini: 106, pl. 25, figs $1-8$.

1990 Macrocyprissa arcuata (Colalongo \& Pasini); Maddocks: 85, figs 12.17-18, 13.17-18, 21.24, 23.24, 24.34, 28.29-30, $29.9,35.19,44.5,47.6-7,50.15-19,51.9-13,56.10,24,27$, $57.11,32,58.18,59.26,33,60.4,63.3,17,64.23,40,51$, $68.5,72.1-3,76.3,78.9-10$; pl. 30, figs $7-10$, pl. 31 , figs 7-11; pl. 67, figs 8-9; pl. 68, figs 6-14; pl. 69, fig. 1; pl. 70, figs $1-4$; pl. 80 , fig. 7 ; pl. 91, figs $10-14$; pl. 104, figs $1-5$; pl. 111 , fig. 6 .

1996 Macrocyprissa arcuata (Colalongo \& Pasini); Coles et al.: 132, pl. 1, figs $10-11$.

Remarks. A comprehensive synonymy list is given in Maddocks (1990)

Superfamily Pontocypridoidea Müller, 1894 Family Pontocyprididae Müller, 1894

Genus Argilloecia Sars, 1866

Type species. Argilloecia cylindrica Sars, 1866
Argilloecia acuminata Müller, 1894

(Fig. 4A-D)

1894 Argilloecia acuminata Müller: 261, pl. 12, figs 1-2, 12-22.

1975 Argilloecia acuminata Müller; Breman: 82, pl. 2, fig. 21, pl. 6, fig. 69.

1987 Argilloecia sp. 5 Whatley \& Coles: 87, pl. 1, figs 19-20.

2004 Argilloecia acuminata Müller; Aiello \& Szczechura: 16, pl. 1 , fig. 2 .

2009b Argilloecia acuminata Müller; Yasuhara et al.: 886, pl. 3, figs 1-2, 4-5.

2009 (part) Argilloecia sp. 2 Alvarez Zarikian: 7, pl. P8, fig. 4 (non 3).

Remarks. Comprehensive synonymy lists are given in Aiello \& Szczechura (2004), Yasuhara et al. (2009b) and supplemented herein.

Argilloecia caju Yasuhara, Okahashi \& Cronin 2009 (Fig. 4E-F)

2009 b Argilloecia сајu Yasuhara, Okahashi \& Cronin: 886, pl. 3, figs $21-24$.

Remarks. Argilloecia caju is similar to Pacific species A. viriosa Hao, 1988 (in Ruan \& Hao, 1988), but distinguished by having a much more slender outline. A. caju was originally reported from the western North Atlantic (Yasuhara et al., 2009b) and is here confirmed in the eastern North Atlantic.

Argilloecia labri sp. nov.

(Fig. 4G-J)

1987 Argilloecia sp. 4 Whatley \& Coles: 86, pl. 1, figs 17-18. 2000 Argilloecia sp. 2 Didié \& Bauch: 116, pl. 3, figs 3-4.

Derivation of name. From Latin labri (noun, genitive singular) $=$ lip.

Diagnosis. A small, moderately calcified Argilloecia species with lip-shaped outline.

Holotype. LV, USNM 603651 (ODP982060) (Fig. 4I).

Paratypes. LV, USNM 603649 (ODP982058); RV, USNM 603650 (ODP982059); RV, USNM 603652 (ODP982061).

Type locality and horizon. ODP 982A, 1/1/50-52.

Description. Carapace moderately calcified, small, highest at midlength. Outline trapezoidal in lateral view; anterior margin rounded and upturned; posterior margin acuminate and slightly upturned; dorsal margin arched; ventral margin slightly sinuous. Anterodorsal and posterodorsal margins obtuse-angular. RV strongly overlaps LV. Lateral surface smooth. Internal features as for genus.

Dimensions. USNM 603651 (ODP982060) (Holotype), L=0.461, $\mathrm{H}=0.202$;

USNM 603650 (ODP982059) (Paratype), $\mathrm{L}=0.497, \mathrm{H}=0.223$.

Remarks. This species is similar to the Pacific species Argilloecia spicata Hao, 1988 (in Ruan \& Hao, 1988), but distinguished by having a much more upturned posterior margin.

Argilloecia bensoni Barra, Aiello \& Bonaduce, 1996

(Fig. 4K-N)

1996 Argilloecia bensoni Barra, Aiello \& Bonaduce: 129, pl. 2, figs $3-4$; pl. 3, figs $1-3$. 


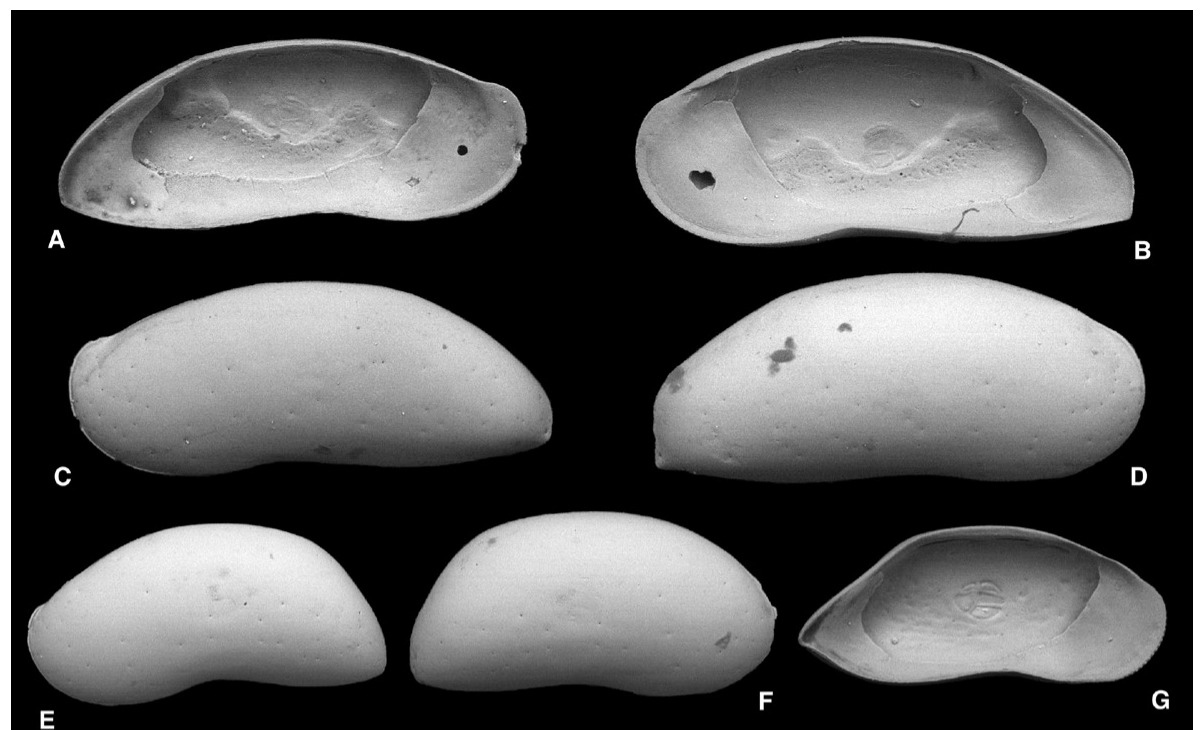

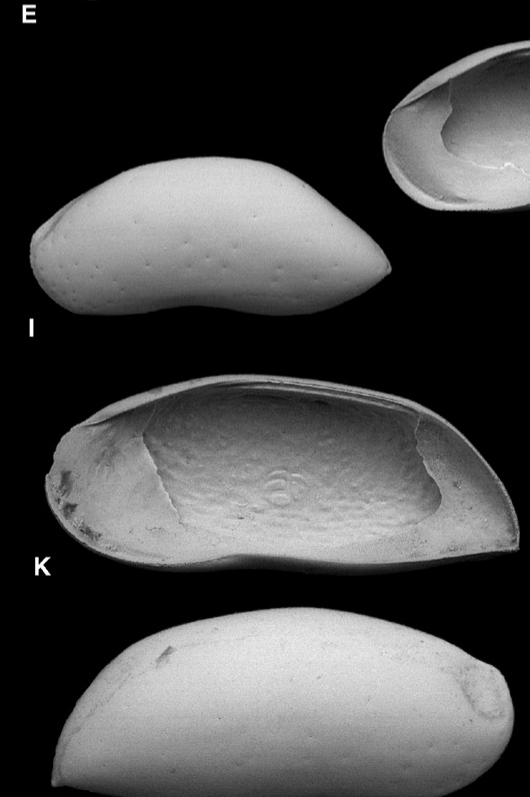

M
H

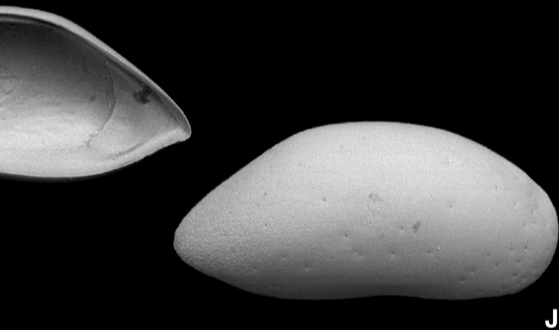

Fig. 4. SEM images of Argilloecia species. A-D, Argilloecia acuminata Müller, 1894: A, USNM 603643 (ODP982062); adult LV from 1/1/60-62; B, USNM 603644 (ODP982063); adult RV from 1/1/60-62; C, USNM 603645 (ODP982064); adult LV from 1/1/50-52; D, USNM 603646 (ODP982065); adult RV from 1/1/50-52. E-F, Argilloecia caju Yasuhara, Okahashi \& Cronin, 2009: E, USNM 603647 (ODP982052); adult LV from 1/2/17-19; F, USNM 603648 (ODP982053); adult RV from 1/2/17-19. G-J, Argilloecia labri sp. nov.: G, Paratype USNM 603649 (ODP982058); adult LV from 1/3/102-104; H, Paratype USNM 603650 (ODP982059); adult RV from 1/3/102-104; I, Holotype USNM 603651 (ODP982060); adult LV from 1/1/50-52; J, Paratype USNM 603652 (ODP982061); adult RV from 1/3/82-84. K-N, Argilloecia bensoni Barra, Aiello \& Bonaduce, 1996: K, USNM 603653 (ODP982054); adult RV from 1/1/97-99; L, USNM 603654 (ODP982057); adult LV from 1/1/142144; M, USNM 603655 (ODP982056); adult RV from 1/3/112-114; N, USNM 603656 (ODP982055); adult LV from 1/1/107-109. C-F, I-J, M-N, lateral views; A-B, G-H, K-L, internal views. All specimens from late Quaternary section of ODP Hole 982A, Rockall Plateau, eastern North Atlantic. Scale bar represents $1 \mathrm{~mm}$.

Remarks. This species was originally reported from Mediterranean Plio-Pleistocene strata.

Genus Propontocypris Sylvester-Bradley, 1947

Type species. Pontocypris trigonella Sars, 1866
Propontocypris acuminata (Müller, 1894)

(Fig. 5A)

1894 Erythrocypris acuminata Müller: 259, pl. 11, figs 5-6, 1618, 40-42; pl. 28, figs 23, 30; pl. 38, figs 47-48.

1976 Pontocypris acuminata (Müller); Bonaduce et al.: 25, pl. 9, figs $1-2$.

2000 Pontocypris sp. Didié \& Bauch: 116, pl. 4, fig. 17. 


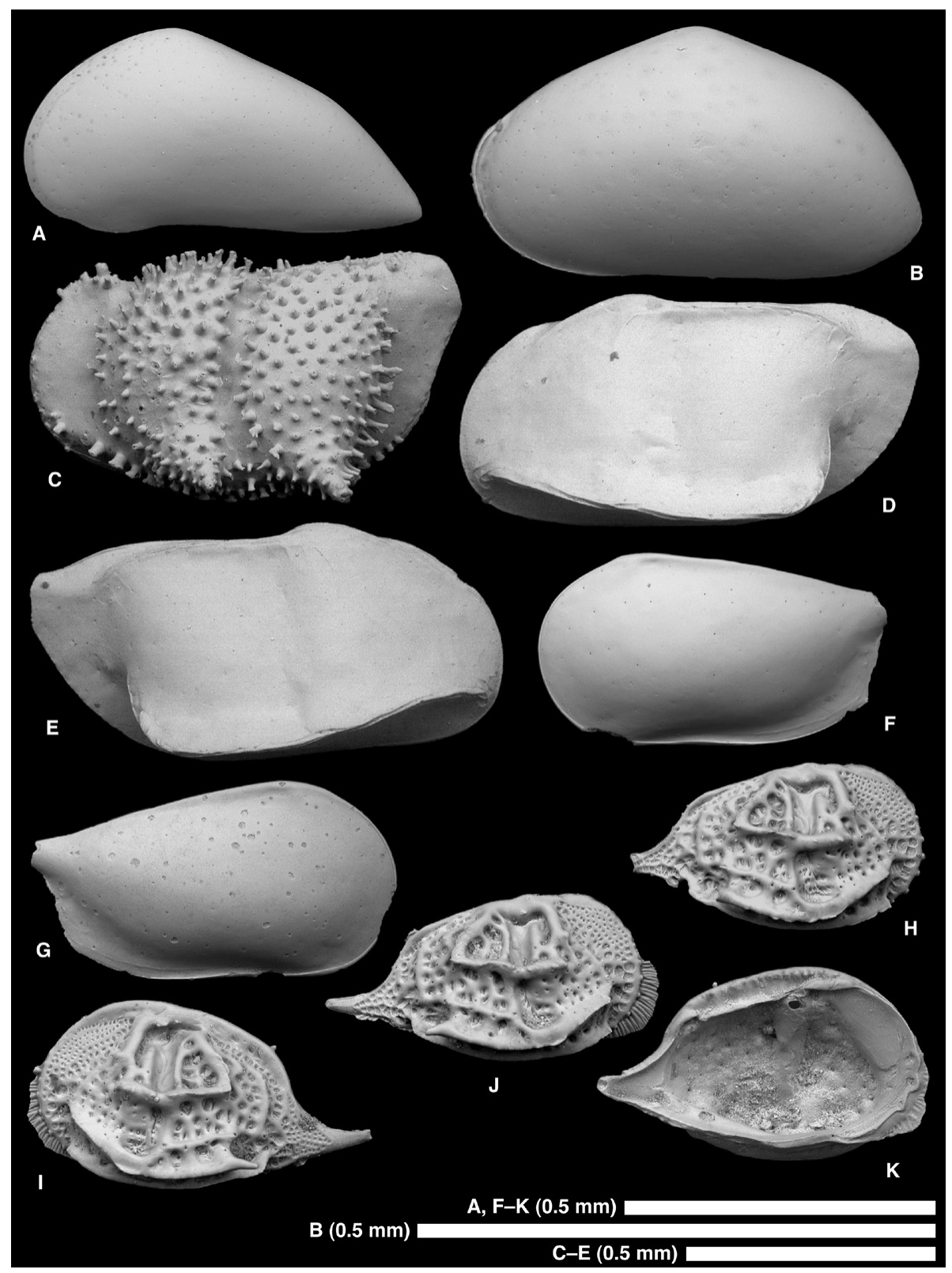

Fig. 5. SEM images of Propontocypris, Bythoceratina, Pseudocythere and Paijenborchella species. A, Propontocypris acuminata (Müller, 1894), USNM 603657 (ODP982165prop); juvenile? LV from 1/2/37-39. B, Propontocypris sp., USNM 603658 (ODP982166prop); juvenile? LV from 1/1/60-62. C, Bythoceratina scaberrima (Brady, 1886), USNM 603659 (ODP982072); adult LV from 1/2/67-69. D-E, Bythoceratina nuda sp. nov.: D, Holotype USNM 603660 (ODP982073); adult LV from 1/3/52-54; E, Paratype USNM 603661 (ODP982074); adult RV from 1/3/42-44. F-G, Pseudocythere caudata Sars, 1866:. F, USNM 603662 (ODP982167pseu); adult? LV from 1/3/42-44; G, USNM 603663 (ODP982168pseu); adult? RV from 1/3/42-44. H-K, Paijenborchella cymbula Ruggieri, 1950: H, USNM 603664 (ODP982131); adult RV from 1/1/97-99; I, USNM 603665 (ODP982132); adult LV from 1/1/40-42; J, USNM 603666 (ODP982133); adult RV from 1/1/40-42; K, USNM 603667 (ODP982134); adult LV from 1/1/40-42. A-J, lateral views; K, internal view. All specimens from late Quaternary section of ODP Hole 982A, Rockall Plateau, eastern North Atlantic. Scale bars represent $0.5 \mathrm{~mm}$.

\section{Propontocypris sp.}

(Fig. 5B)

?2009 Propontocypris trigonella Sars; Alvarez Zarikian: 7, pl. P8, fig. 10 .

Suborder Cytherocopina Gründel, 1967 Superfamily Cytheroidea Baird, 1850
Family Bythocytheridae Sars, 1866

Genus Bythoceratina Hornibrook, 1952

Type species. Bythoceratina mestayerae, Hornibrook, 1952

\section{Bythoceratina scaberrima (Brady, 1886)}

(Fig. 5C)

1886 Cytherura scaberrima Brady: 198, pl. 14, figs 10-11. 
1980 Bythoceratina scaberrima mediterranea Colalongo \& Pasini:

72, pl. 1, fig. 9; pl. 4, figs 9-10.

2001 Bythoceratina scaberrima (Brady); Didié \& Bauch: pl. 1,

fig. 29 (erratum for Didié \& Bauch, 2000).

2005 Retibythere scaberrima (Brady); Guernet: 109.

Remarks. Comprehensive synonymy list and detailed discussion in Guernet (2005) and Yasuhara et al. (in press b).

\section{Bythoceratina nuda sp. nov.}

(Fig. 5D-E)

Derivation of name. From Latin nuda (adjective, nominative singular, gender feminine or neuter) $=$ stripped, with reference to its carapace without any spine or reticulation.

Diagnosis. A large, moderately calcified Bythoceratina species without spines or reticulation.

Holotype. LV, USNM 603660 (ODP982073) (Fig. 5D)

Paratype. RV, USNM 603661 (ODP982074).

Type locality and horizon. ODP 982A, 1/3/52-54.

Description. Carapace moderately calcified, large, highest at anterodorsal corner (=anterior cardinal angle). Outline parallelogram-like in lateral view; anterior margin rounded; caudal process upturned; dorsal margin sinuous; ventral margin slightly curved. Anterodorsal margin prominent; posterodorsal margin slightly angular. Lateral surface smooth. A ventrolateral ridge well developed, reaching to anterior margin; thin dorsolateral ridge present. A median sulcus present, but very shallow. Internal features as for genus.

Dimensions. USNM 603660 (ODP982073) (Holotype), L=0.920, $\mathrm{H}=0.462$; USNM 603661 (ODP982074) (Paratype), $\mathrm{L}=0.948$, $\mathrm{H}=0.471$.

Remarks. This species is distinguished from any other Bythoceratina species by its lack of spines and reticulation.

Genus Pseudocythere Sars, 1866

Type species. Pseudocythere caudata Sars, 1866

Pseudocythere caudata Sars, 1866

(Fig. 5F-G)

1866 Pseudocythere caudata Sars: 88.

1926 Pseudocythere caudata Sars; Sars: 239, pl. 109, fig. 2a-k.

2009 b Pseudocythere caudata Sars; Yasuhara et al.: 892, pl. 4, figs $7-12$.

Remarks. We think that this species has considerable intraspecific variation. A comprehensive synonymy list and detailed discussion are given in Yasuhara et al. (in press $b$ ).

Family Cytheridae Baird, 1850

Genus Paijenborchella Kingma, 1948

Type species. Paijenborchella iocosa Kingma, 1948

Paijenborchella cymbula Ruggieri, 1950

(Fig. 5H-K)

1950 Paijenborchella cymbula Ruggieri: 60, 1 unnumbered fig. on p. 61.
1973 Paijenborchella (Eopaijenborchella) malaiensis cymbula Ruggieri; Doruk: 161, pls 1.30.162, 1.30.164.

2000 Paijenborchella malaiensis cymbula Ruggieri; Aiello et al.: 93, pl. 2, fig. 12.

2005 Paijenborchella cymbula Ruggieri; Guernet: 107.

Remarks. A comprehensive synonymy list can be found in Aiello et al. (2000) and Guernet (2005). To our knowledge, this is the first well-illustrated record (SEM images) of this species from the Atlantic. This species was recently reported from the Iberian Margin at IODP Site U1387 (Expedition 339 Scientists, 2013).

Family Cytheruridae Müller, 1894

Genus Aversovalva Hornibrook, 1952

Type species. Cytheropteron

(Aversovalva) aureum Hornibrook, 1952

Remarks. Several authors have considered Aversovalva Hornibrook, 1952 as a subgenus (Aiello et al., 1996a) or junior synonym (Whatley \& Masson, 1979; Horne \& Whittaker, 1988) of Cytheropteron Sars, 1866. However, in our opinion, differences in hingement and outline including a straight median hinge element, highly developed smooth and rounded terminal teeth clearly distinct from the median hinge element, and parallelogram-like outline in Aversovalva (e.g. Figs 6C-D) are sufficient to separate Aversovalva from Cytheropteron. Most recent deep-sea ostracod papers have considered Aversovalva as an independent genus (e.g. see synonymy of Aversovalva hydrodynamica below), supporting our decision.

\section{Aversovalva hydrodynamica Whatley \& Coles, 1987}

(Fig. 6A-D)

1987 Aversovalva hydrodynamica Whatley \& Coles: 69, pl. 3, figs $10-11$.

1996 Aversovalva hydrodynamica Whatley \& Coles; Coles et al.: 150, pl. 3, fig. 17.

1988 (part) Aversovalva sp. 2 Whatley \& Ayress: 742, pl. 2, fig. 1a (non 1b).

?2001 Aversovalva sp. cf. A. hydrodynamica Didié \& Bauch: 103, pl. 1, fig. 12 (as erratum for Didié \& Bauch, 2000).

2009 Aversovalva hydrodynamica Whatley \& Coles; Alvarez Zarikian: 3, pl. P3, fig. 7 .

Remarks. Very similar, but slightly different species are reported from the western North Atlantic as Aversovalva sp. 1 and $A$. cf. hydrodynamica (Yasuhara et al., 2009b). A Pliocene Mediterranean species Aversovalva denticulatum (Aiello, Barra \& Bonaduce, 1996) shows strong affinity to A. hydrodynamica Whatley \& Coles, 1987, but the former has a more triangular outline. $A$. hydrodynamica is also similar to A. consueta (Dall'Antonia, 2003), but the latter has thicker and more downward-extended alae and weaker reticulation. Although Coles et al. (1990, 1996) suggested a global distribution for this species, reliable records with SEM images are restricted in the eastern North Atlantic.

Genus Cytheropteron Sars, 1866

Type species. Cythere latissima Norman, 1865 (designated by Brady \& Norman, 1889; see Horne \& Whittaker (1988) for details and lectotype).

Remarks. We agree with Horne \& Whittaker (1988) and consider Kobayashiina Hanai, $1957 b$ and Lobosocytheropteron Ishizaki \& Gunther, 1974 as junior synonyms of Cytheropteron. 


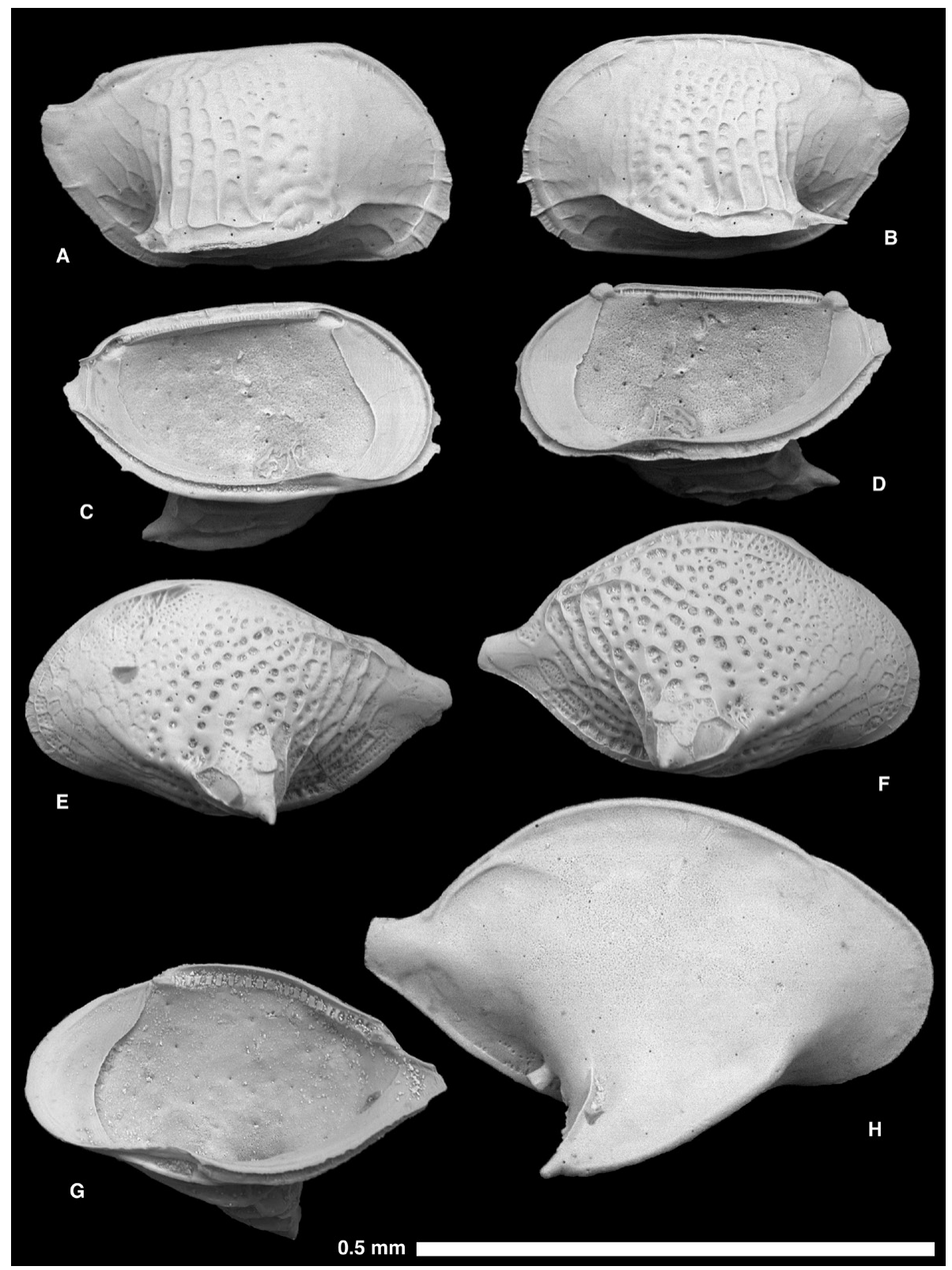

Fig. 6. SEM images of Aversovalva and Cytheropteron species. A-D, Aversovalva hydrodynamica Whatley \& Coles, 1987: A, USNM 603668 (ODP982048); adult RV from 1/1/107-109; B, USNM 603669 (ODP982049); adult LV from 1/1/107-109; C, USNM 603670 (ODP982050); adult LV from 1/1/107-109; D, USNM 603671 (ODP982051); adult RV from 1/1/117-119. E-G, Cytheropteron aielloi Yasuhara, Okahashi \& Cronin, 2009: E, USNM 603672 (ODP982042); adult LV from 1/3/42-44; F, USNM 603673 (ODP982043); adult RV from 1/3/42-44; G, USNM 603674 (ODP982044); adult RV from 1/3/42-44; H, Cytheropteron alatum Sars, 1866, USNM 603675 (ODP982084); adult RV from 1/3/112-114. A-B, E-F, H, lateral views; C-D, G, internal views. All specimens from late Quaternary section of ODP Hole 982A, Rockall Plateau, eastern North Atlantic. Scale bar represents $0.5 \mathrm{~mm}$.

Cytheropteron aielloi Yasuhara, Okahashi \& Cronin, 2009

(Fig. 6E-G)

1996 Cytheropteron sedovi Schneider; Whatley et al.: 19, pl. 2, figs $15-17$.

1998 Cytheropteron sedovi Schneider; Whatley et al.: 21, pl. 2, figs $11-12$.

2009 Cytheropteron aielloi Yasuhara, Okahashi \& Cronin: 898, pl. 10, figs 3-6.

Remarks. This species is known not only from the North Atlantic proper but also from the Nordic seas.

\section{Cytheropteron alatum Sars, 1866}

(Fig. 6H)

1866 Cytheropteron alatum Sars: 81.

1926 Cytheropteron alatum Sars; Sars: 225, pl. 104, fig. 1.

1993 Cytheropteron alatum Sars; Penney: figs 4n-o.

1996 Cytheropteron vespertilio (Reuss); Coles et al.: 136, pl. 3, fig. 9.

1998 Cytheropteron alatum Sars; Freiwald \& Mostafawi: 260, pl. 59, fig. 7.

2000 Cytheropteron alatum Sars; Didié \& Bauch: pl. 2, fig. 6. 
Remarks. Reliable occurrence records of this species with SEM image(s) or sketches are known only from the eastern North Atlantic as listed in the synonymy list above. Detailed discussion of this species can be found in Yasuhara et al. (in press $b$ ).

Cytheropteron colesoabyssorum sp. nov.

(Fig. 7A)

1996 Cytheropteron cf. abyssorum Brady; Coles et al.: 136, pl. 3, figs $12-13$.

Derivation of name. In honour of Graham P. Coles for his contribution to deep-sea ostracod research; and with reference to its similarity to Cytheropteron abyssorum as indicated by him.

Diagnosis. A large, moderately calcified Cytheropteron species with finely punctate carapace, upturned caudal process, and relatively rounded outline.

Holotype. RV, USNM 603676 (ODP982077) (Fig. 7A).

Type locality and horizon. ODP 982A, 1/2/37-39.

Description. Carapace moderately calcified, large, highest at midlength. Outline rhomboidal and rounded in lateral view; anterior margin evenly rounded; caudal process strongly upturned; dorsal margin arched; ventral margin slightly curved; alae well developed, almost reaching to anterior margin and slightly extended below ventral margin; median sulcus present on alae; thin dorsolateral ridge present along dorsal margin. Anterodorsal and posterodorsal margins slightly angular. Lateral surface finely punctate. Internal features as for genus.

Dimensions. USNM 603676 (ODP982077) (Holotype), L=0.489, $\mathrm{H}=0.301$.

Remarks. Cytheropteron colesoabyssorum sp. nov. is similar to C. abyssorum Brady, 1880 (see Passlow \& Ayress, 1994) in certain aspects, such as presence of a median sulcus on alae and punctate carapace, but easily distinguished by having much finer punctation covering entire carapace, thinner and longer alae, and upturned caudal process and presence of dorsolateral ridge. C. colesoabyssorum sp. nov. is similar to $C$. cf. tenuialatum of Coles et al. (1996), but distinguished by having a dorsolateral ridge and more strongly upturned caudal process, and by the absence of primary reticulation on the caudal process.

\section{Cytheropteron colesopunctatum sp. nov.}

(Fig. 7B-C)

1996 (part) Cytheropteron gr. punctatum Brady; Coles et al.: 136, pl. 3, figs 7-8 (non 5-6).

in press a Cytheropteron sp. Yasuhara et al.: fig. 6.7-8.

Derivation of name. In honour of Graham P. Coles for his contribution to deep-sea ostracod research; and with reference to its similarity to Cytheropteron punctatum as indicated by him.

Diagnosis. A large, moderately calcified Cytheropteron species with coarsely punctate carapace and horizontally long, well-developed alae.

Holotype. RV, USNM 603677 (ODP982082) (Fig. 7B).

Paratype. LV, USNM 603678 (ODP982083).

Type locality and horizon. ODP 982A, 1/1/30-32.
Description. Carapace moderately calcified, large, highest at midlength. Outline rhomboidal or almond-like in lateral view; anterior margin evenly rounded; caudal process prominent and upturned; dorsal margin arched and slightly sinuous; ventral margin curved; alae curved, horizontally long and well-developed, reaching to anterior margin and extending slightly below ventral margin, with a small spine at apex. Anterodorsal margin slightly angular; posterodorsal margin weakly angular. Lateral surface coarsely punctate; primary and secondary reticulation developed in posterior one third. Internal features as for genus.

Dimensions. USNM 603677 (ODP982082) (Holotype), L=0.514, $\mathrm{H}=0.306$; USNM 603678 (ODP982083) (Paratype), $\mathrm{L}=0.545$, $\mathrm{H}=0.338$.

Remarks. Cytheropteron colesopunctatum sp. nov. is similar to C. punctatum Brady, 1868 in certain aspects, such as punctate carapace and general outline, but is distinguished by having smoothly curved and horizontally longer alae reaching to anterior margin and well-developed primary and secondary reticulation in posterior one-third. C. punctatum has sinuous and horizontally shorter alae and no or only poorly developed reticulation, according to the sketches and SEM images shown in Brady (1868), Sars (1928) and Whatley \& Masson (1979). C. colesopunctatum sp. nov. is similar to $C$. paracarolinae Zhao et al., 2000 (see Zhao et al., 2000; Hou \& Gou, 2007), but the latter is larger, lacks punctation in anterior one-third, and has more slender outline and stronger caudal process.

Cytheropteron didieae Yasuhara, Okahashi \& Cronin 2009 (Fig. 7D-H)

2009 Cytheropteron didieae Yasuhara, Okahashi \& Cronin: 900, pl. 6, figs 5-6, 8-9, 11-12.

Remarks. This is the first record of the species from the eastern North Atlantic.

Cytheropteron fugu Yasuhara, Okahashi \& Cronin, 2009

(Figs. 7I-J, 8A-B)

2009 Cytheropteron fugu Yasuhara, Okahashi \& Cronin: 902, pl. 7, figs 1-6.

Remarks. This is the first record of the species from the eastern North Atlantic.

Cytheropteron inornatum Brady \& Robertson, 1872

(Fig. 8G-J)

1872 Cytheropteron inornatum Brady \& Robertson: 61, pl. 2, figs 1-3.

1979 Cytheropteron inornatum Brady \& Robertson; Whatley \& Masson: 238, pl. 3, figs 1-3, 5-7.

1981 Cytheropteron hanaii Ishizaki: 55, pl. 11, figs 11-12; pl. 12, figs $1-4$; pl. 13 , figs $8-9$; pl. 14, fig. 3 .

1988 Cytheropteron hanaii Ishizaki; Wang et al.: 265, pl. 52, figs 17-18.

1989 Cytheropteron inornatum Brady \& Robertson; Athersuch et al.: 226, fig. 95; pl. 8, fig. 4.

1992 Cytheropteron hanaii Ishizaki; Ikeya \& Suzuki: 126, pl. 4, fig. 4.

1996 Cytheropteron inornatum Brady \& Robertson; Coles et al: 135, pl. 2, fig. 15 . 


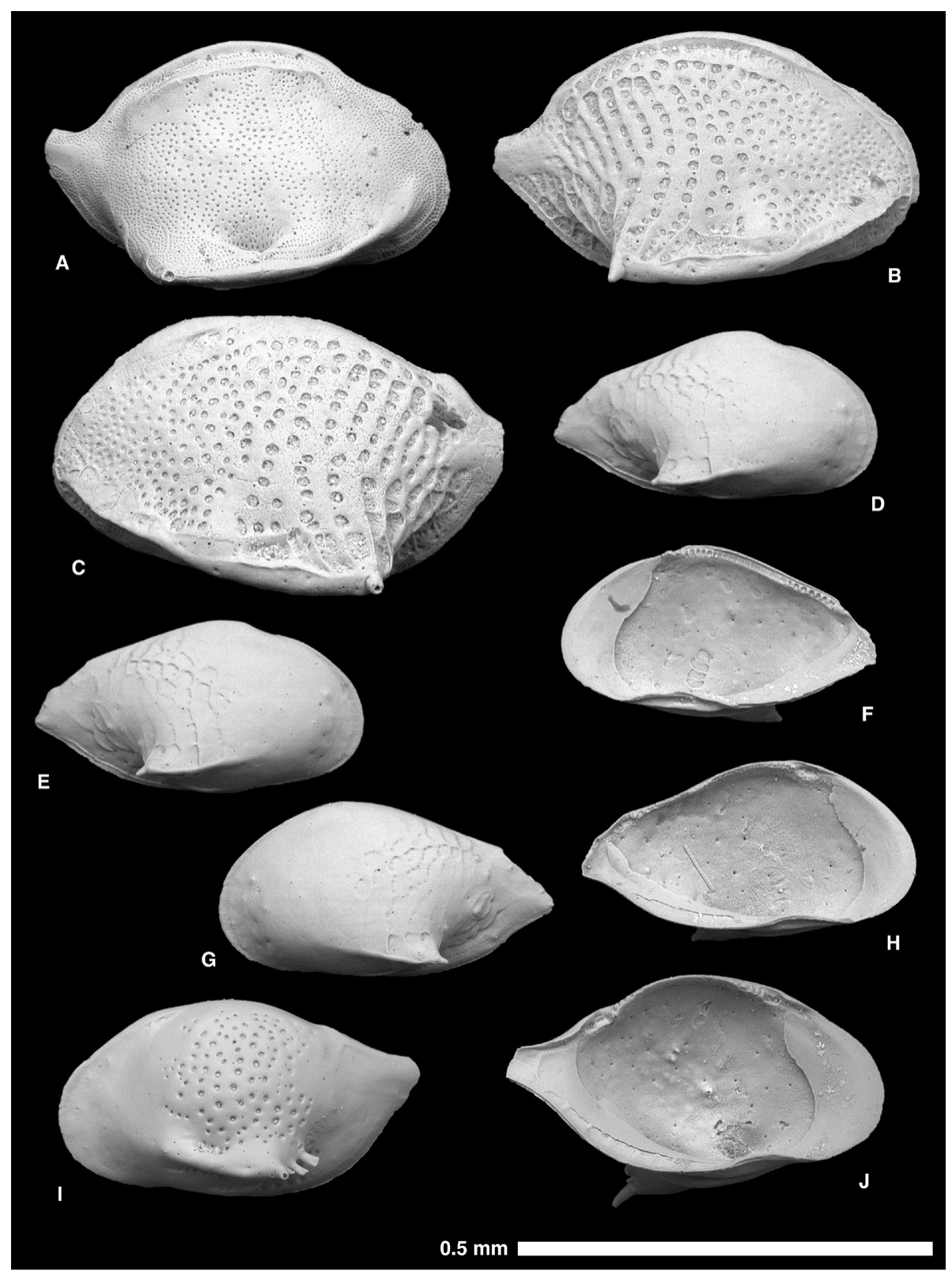

Fig. 7. SEM images of Cytheropteron species. A, Cytheropteron colesoabyssorum sp. nov., Holotype USNM 603676 (ODP982077); adult RV from 1/2/37-39. B-C, Cytheropteron colesopunctatum sp. nov.: B, Holotype USNM 603677 (ODP982082); adult RV from 1/1/30-32; C, Paratype USNM 603678 (ODP982083); adult LV from 1/1/30-32. D-H, Cytheropteron didieae Yasuhara, Okahashi \& Cronin, 2009: D, USNM 603679 (ODP982085); adult RV from 1/1/50-52; E, USNM 603680 (ODP982086); adult RV from 1/1/60-62; F, USNM 603681 (ODP982087); adult RV from 1/1/60-62; G, USNM 603682 (ODP982088); adult LV from 1/1/60-62; H, USNM 603683 (ODP982089); adult LV from 1/2/127-129. I-J, Cytheropteron fugu Yasuhara, Okahashi \& Cronin, 2009: I, USNM 603684 (ODP982035); adult LV from 1/2/67-69; J, USNM 603685 (ODP982036); adult LV from 1/2/67-69. A-E, G, I, lateral views; F, H, J, internal views. All specimens from late Quaternary section of ODP Hole 982A, Rockall Plateau, eastern North Atlantic. Scale bar represents $0.5 \mathrm{~mm}$.

2000 Cytheropteron hanaii Ishizaki; Zhao et al:: 262, pl. 3, figs $8-9$.

2007 Cytheropteron hanaii Ishizaki; Hou \& Gou: 294, pl. 119, figs $14-15$; pl. 122, figs $11-12$.

2009 Cytheropteron sp. g Yasuhara, Okahashi \& Cronin: 908, pl. 6, fig. 14.

2012 Cytheropteron hanaii Ishizaki; Tanaka et al.: 10, pl. 1, fig. 13.
Remarks. Detailed comparison with similar species such as C. fraudulentum Aiello, Barra \& Bonaduce 1996 and C. sulcatum Bonaduce, Ciampo \& Masoli, 1976 is found in Aiello et al. (1996a) and Athersuch et al. (1989). In our opinion, C. hanaii Ishizaki, 1981 (see Ishizaki, 1981; Zhao et al., 2000; Hou \& Gou, 2007 ) is a junior synonym of $C$. inornatum Brady \& Robertson, 1872. 


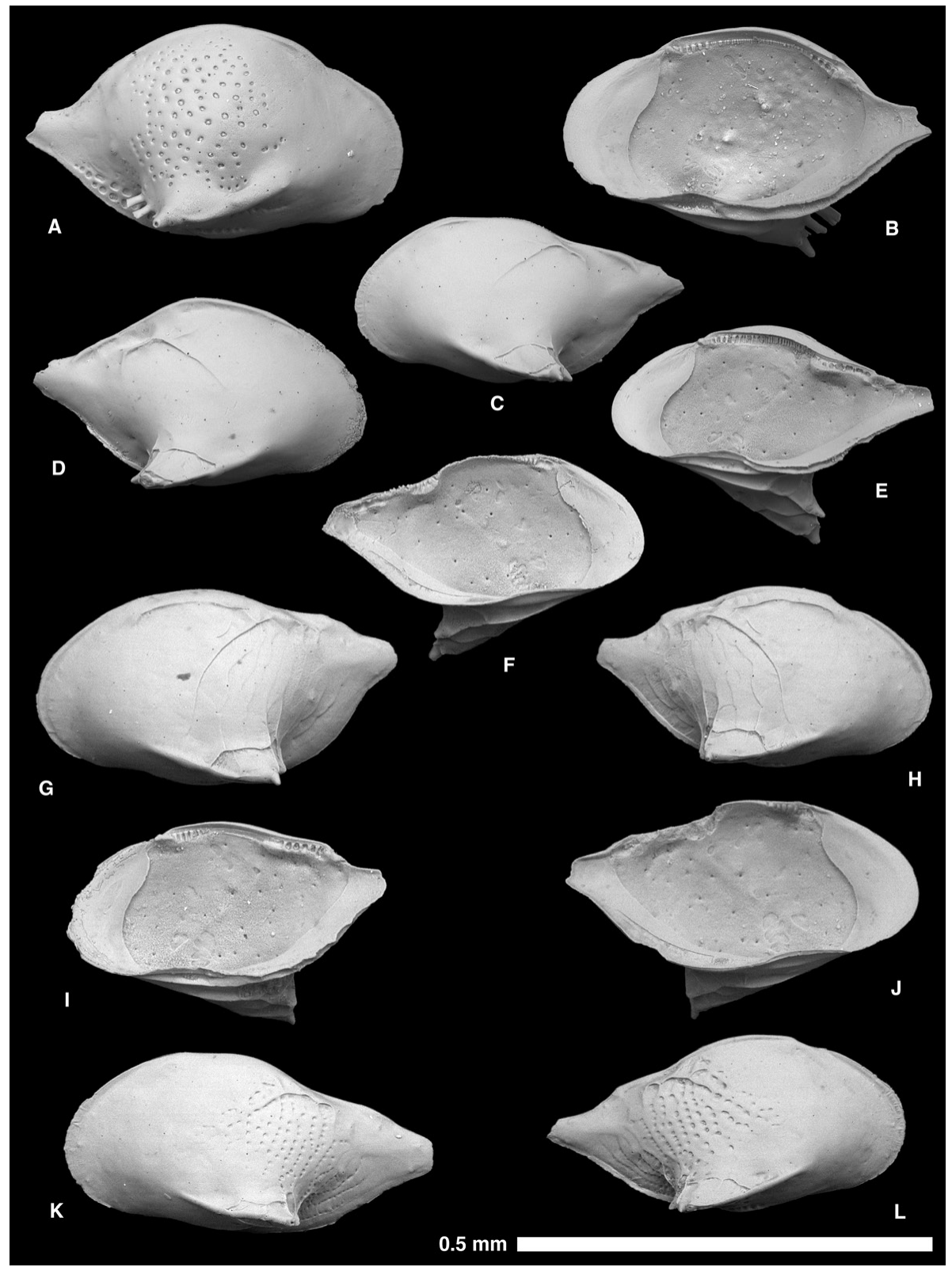

Fig. 8. SEM images of Cytheropteron species. A-B, Cytheropteron fugu Yasuhara, Okahashi \& Cronin, 2009: A, USNM 603686 (ODP982037); adult RV from 1/2/57-59; B, USNM 603687 (ODP982038); adult RV from 1/2/57-59. C-F, Cytheropteron omega Aiello, Barra \& Bonaduce, 1996: C, USNM 603688 (ODP982040); adult LV from 1/4/12-14; D, USNM 603689 (ODP982039); adult RV from 1/4/12-14; E, USNM 603690 (ODP982041); adult RV from 1/3/132-134; F, USNM 603691 (ODP982045); adult LV from 1/2/127-129. G-J, Cytheropteron inornatum Brady \& Robertson, 1872: G, USNM 603692 (ODP982080); adult LV from 1/1/97-99; H, USNM 603693 (ODP982079); adult RV from 1/2/17-19; I, USNM 603694 (ODP982081); adult RV from 1/1/97-99; J, USNM 603695 (ODP982078); adult LV from 1/2/17-19. K-L, Cytheropteron massoni Whatley \& Coles, 1987: K, USNM 603696 (ODP982047); adult LV from 1/1/117-119; L, USNM 603697 (ODP982046); adult RV from 1/2/17-19. A, C-D, G-H, K-L, lateral views; B, E-F, I-J, internal views. All specimens from late Quaternary section of ODP Hole 982A, Rockall Plateau, eastern North Atlantic. Scale bar represents $0.5 \mathrm{~mm}$.

\section{Cytheropteron massoni Whatley \& Coles, 1987}

(Fig. 8K-L)

1987 Cytheropteron massoni Whatley \& Coles: 63, pl. 2, figs 15-17. 2000 Cytheropteron massoni Whatley \& Coles; Didié \& Bauch: 113, pl. 2, fig. 11.

2009 b Cytheropteron massoni Whatley \& Coles; Yasuhara et al.: 904, p. 6, figs 7, 10, 13.
Remarks. C. massoni Whatley \& Coles, 1987 is known from both the eastern and western North Atlantic.

\section{Cytheropteron omega Aiello, Barra \& Bonaduce, 1996} (Fig. 8C-F)

1987 (part) Cytheropteron syntomoalatum Whatley \& Coles: pl. 2, fig. 27 (non pl. 2, figs 25-26, 28-29). 
1996 Cytheropteron omega Aiello, Barra \& Bonaduce: 170, pl. 2, figs 7-9.

Remarks. Detailed comparison with similar species such as C. garganicum Bonaduce, Ciampo \& Masoli, 1976 can be found in Aiello et al. (1996a). Our specimens have relatively weakly developed dorsal ridges compared to the type specimens and thus the 'upside-down omega' structure is unclear, but otherwise identical. We consider this difference as intraspecific variation. Wellpreserved specimens shown here indicate that there are two spines at the apex of alae. A paratype specimen of C. syntomoalatum of Whatley \& Coles (1987, pl. 2, fig. 27) is not conspecific with C. syntomoalatum Whatley \& Coles, 1987 and is considered here and by Aiello et al. (1996a) as C. omega Aiello, Barra \& Bonaduce, 1996, although this specimen has only one spine at the apex of the alae and a slightly more slender outline.

\section{Cytheropteron paramediotumidum sp. nov.}

(Fig. 9A-B)

1996 (part) Cytheropteron gr. punctatum Brady; Coles et al.: 136, pl. 3, figs 5-6 (non 7-8).

Derivation of name. With reference to its similarity to Cytheropteron mediotumidum.

Diagnosis. A large, moderately calcified Cytheropteron species with finely punctate carapace and straight-sided alae.

Holotype. LV, USNM 603698 (ODP982092) (Fig. 9A).

Paratype. RV, USNM 603699 (ODP982093).

Type locality and horizon. ODP 982A, 1/2/107-109.

Description. Carapace moderately calcified, large, highest at midlength. Outline subrhomboidal in lateral view; anterior margin rounded; caudal process moderately prominent; dorsal margin arched; ventral margin slightly curved; alae straight, thin and horizontally long, almost reaching to anterior margin, and extending slightly below ventral margin; a small subcentral depression present on alae. Anterodorsal margin slightly angular; posterodorsal margin weakly prominent. Lateral surface finely punctate in posterior two-thirds; primary and secondary reticulation weakly developed in posterior one-third. Internal features as for genus.

Dimensions. USNM 603698 (ODP982092) (Holotype), L =0.638, $\mathrm{H}=0.389$; USNM 603699 (ODP982093) (Paratype), $\mathrm{L}=0.671$, $\mathrm{H}=0.431$.

Remarks. Cytheropteron paramediotumidum sp. nov. is very similar to C. mediotumidum Zhao, Whatley \& Zhou, 2000, but distinguished by having a less upturned caudal process, finer punctation, less distinct primary reticulation and straight-sided alae. C. paramediotumidum sp. nov. is also similar to $C$. colesopunctatum sp. nov., but the latter has more prominent caudal processes, coarser and more distinct punctation, and curved-sided alae.

Cytheropteron demenocali Yasuhara, Okahashi \& Cronin, 2009 (Fig. 9C-D)

?2000 Cytheropteron porterae Whatley \& Coles; Didié \& Bauch: 113, pl. 2, fig. 20 (non figs 19 and 21).

2009 Cytheropteron demenocali Yasuhara, Okahashi \& Cronin: 900 , pl. 9 , figs $1-10$.
Remarks. Our specimens have two spines (instead of one) at the apex of alae and slightly more ventrally-extended alae, but are otherwise identical. We consider these differences as intraspecific variation, at least for now.

Cytheropteron pararhombiformis Zhao, Whatley \& Zhou, 2000 (Fig. 9E-F)

1988 Cytheropteron rhombiformis Chen; Ruan \& Hao: 283, pl. 47, figs $18-20$.

1996 Cytheropteron sp. Coles et al.: 136, pl. 3, fig. 16.

2000 Cytheropteron pararhombiformis Zhao, Whatley \& Zhou: 275, pl. 4, figs 5-8.

2007 Cytheropteron pararhombiforme Zhao, Whatley \& Zhou; Hou \& Gou: 301, pl. 123, figs 5-8.

Remarks. Cytheropteron pararhombiformis Zhao, Whatley \& Zhou, 2000 is very similar to C. zinzulusae Bonaduce, Ciampo \& Masoli, 1976, but distinguished by having irregular and coarser punctation and a less upturned caudal process. This species was originally reported from the western North Pacific.

\section{Cytheropteron paucipunctatum Whatley \& Coles, 1987}

(Fig. 9G-H)

1987 Cytheropteron paucipunctatum Whatley \& Coles: 63, pl. 2, figs $18-20$.

1988 Cytheropteron sp. 1 Whatley \& Ayress: 740, pl. 1, fig. 6a-b.

Remarks. Our specimens have weakly developed primary reticulation in the posterior one-third, but otherwise are identical to C. paucipunctatum Whatley \& Coles, 1987. We consider this difference as intraspecific variation. This species is known only from the eastern North Atlantic.

Cytheropteron perlaria Hao, 1988 (in Ruan \& Hao, 1988)

(Fig. 10A-B)

1988 Cytheropteron perlaria Hao (in Ruan \& Hao, 1988): 280, pl. 47, figs 4-9.

1996 Cytheropteron testudo Sars; Coles et al.: 136, pl. 3, figs $10-11$.

1999 Cytheropteron perlaria Hao; Swanson \& Ayress: 155, pl. 1, figs 7-13; pl. 2, figs $1-3$.

non 2004 Cytheropteron perlaria Hao; Ayress et al:: 29, pl. 3, figs $7-8$.

2006 Cytheropteron perlaria Hao; Stepanova: S163, pl. 3, figs $8-10$.

2007 Cytheropteron testudo Sars; Hou \& Gou: 290, pl. 120, figs 9-10.

2009 Cytheropteron perlaria Hao; Alvarez Zarikian: 4, pl. P3, figs $1-2$.

2009 b Cytheropteron perlaria Hao; Yasuhara et al.: 904, pl. 7, figs $12-13$.

2011 Cytheropteron perlaria Hao; Zhao et al:: 27, pl. 1, fig. 26.

Remarks. This species is known from the Atlantic, Arctic and Pacific oceans.

Cytheropteron pherozigzag Whatley, Ayress \& Downing, 1986 (Fig. 10C-D)

1986 Cytheropteron pherozigzag Whatley, Ayress \& Downing: 32, pl. 1, figs 6-20. 


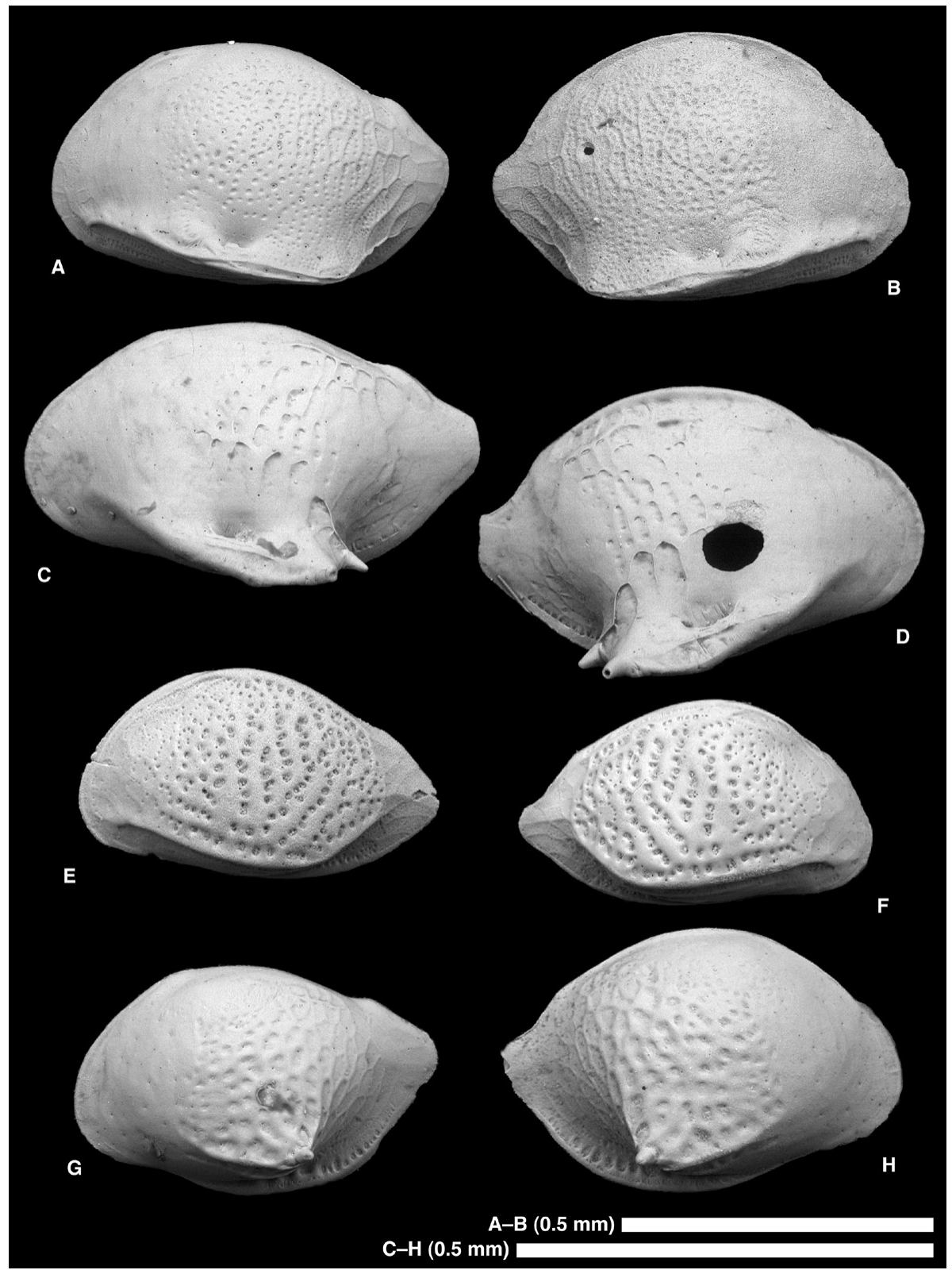

Fig. 9. SEM images of Cytheropteron species. A-B, Cytheropteron paramediotumidum sp. nov.: A, Holotype USNM 603698 (ODP982092); adult LV from 1/2/107-109; B, Paratype USNM 603699 (ODP982093); adult RV from 1/2/127-129. C-D, Cytheropteron demenocali Yasuhara, Okahashi \& Cronin, 2009: C, USNM 603700 (ODP982090); adult LV from 1/1/137-139; D, USNM 603701 (ODP982091); adult RV from 1/2/17-19. E-F, Cytheropteron pararhombiformis Zhao, Whatley \& Zhou, 2000: E, USNM 603702 (ODP982095); adult LV from 1/1/30-32; F, USNM 603703 (ODP982096); adult RV from 1/1/40-42. G-H, Cytheropteron paucipunctatum Whatley \& Coles, 1987: G, USNM 603704 (ODP982097); adult LV from 1/2/127-129; H, USNM 603705 (ODP982098); adult RV from 1/3/52-54. All lateral views. All specimens from late Quaternary section of ODP Hole 982A, Rockall Plateau, eastern North Atlantic. Scale bars represent $0.5 \mathrm{~mm}$.

1988 Cytheropteron pherozigzag Whatley, Ayress \& Downing;

Whatley \& Ayress: pl. 2, fig. 3a-b.

1996 Cytheropteron pherozigzag Whatley, Ayress \& Downing; Zhao \& Zheng: 72, pl. 2, fig. 3.

2000 Cytheropteron pherozigzag Whatley, Ayress \& Downing; Zhao et al.: 263, pl. 1, fig. 20.

2005 Cytheropteron pherozigzag Whatley, Ayress \& Downing; Zhao: 39, pl. 2, fig. 15.
2007 Lobosocytheropteron pherozigzag (Whatley, Ayress \& Downing); Hou \& Gou: 309, pl. 125, fig. 17.

2009 b Cytheropteron pherozigzag Whatley, Ayress \& Downing; Yasuhara et al.: 906, pl. 5, figs 6-8, 10.

Remarks. This species is known from the eastern and western North Atlantic and northwestern Pacific oceans. 
Cytheropteron pseudoalatum Colalongo \& Pasini, 1980

(Fig. 10E)

1980 Cytheropteron pseudoalatum Colalongo \& Pasini: 92, pl. 8, fig. 8; pl. 9, figs $1-5$.

1996 a Cytheropteron pseudoalatum Colalongo \& Pasini; Aiello et al.: 171, pl. 2, figs 1-3; pl. 3, figs 1-2.

Remarks. This species was originally reported from the Mediterranean. This is the first record from the North Atlantic.

Genus Eucytherura Müller, 1894

Type species. Cythere complexa Brady, 1867 (designated by Alexander, 1936).

Remarks. We agree with Ayress et al. (1995) and consider Typhlocythere Bonaduce, Ciampo \& Masoli, 1976, Typhloeucytherura Colalongo \& Pasini, 1980 and Parahemingwayella Dingle, 1984 as junior synonyms of Eucytherura Müller, 1894.

Eucytherura calabra (Colalongo \& Pasini, 1980)

(Fig. 10F-H)

1980 Typhloeucytherura calabra Colalongo \& Pasini: 122, pl. 20, figs $1-8$; pl. 21, figs $1-2$.

1987 Eucytherura calabra (Colalongo \& Pasini); Whatley \& Coles: pl. 3, figs 14-16.

1988 Eucytherura sp. 1; Ruan \& Hao: 291, pl. 49, fig. 18.

1988 Eucytherura calabra (Colalongo \& Pasini); Whatley \&

Ayress: pl. 1, fig. 9a-b.

1995 Eucytherura calabra (Colalongo \& Pasini); Ayress et al: 211, fig. 3A-D.

1996 Eucytherura calabra (Colalongo \& Pasini); Coles et al.: 136, pl. 3, fig. 18 .

1996 Eucytherura calabra (Colalongo \& Pasini); Zhao \& Zheng:

72 , pl. 2, fig. 36 .

2001 Eucytherura calabra (Colalongo \& Pasini); Didié \& Bauch

(as erratum of Didié \& Bauch, 2000): 103, pl. 1, figs 9-10.

Remarks. Eucytherura calabra (Colalongo \& Pasini, 1980) is similar to E. spinicorona Yasuhara, Okahashi \& Cronin 2009, but the former has a curved dorsal margin and rectangular outline. E. calabra is known from the Atlantic, Mediterranean and Pacific.

\section{Eucytherura multituberculata Ayress, Whatley, Downing \& Millson, 1995 \\ (Fig. 10I-J)}

1983 ?Tuberculocythere sp. Cronin: 107, pl. 6, fig. A.

1987 Eucytherura sp. 2 Whatley \& Coles: 90, pl. 3, fig. 18. 1995 Eucytherura multituberculata Ayress, Whatley, Downing \& Millson: 213, fig. 5A-E.

Remarks. This species is known both from the western and eastern North Atlantic.

Eucytherura tetrapteron (Bonaduce, Ciampo \& Masoli, 1976)

(Fig. $11 \mathrm{~A}-\mathrm{C})$

1976 ?Cytheropteron tetrapteron Bonaduce, Ciampo \& Masoli: 99 , pl. 47, fig 1-7.

1980 Cytheropteron? tetrapteron Bonaduce, Ciampo \& Masoli; Ciampo: 19, pl. 3, fig. 5 .

1980 Tuberculocythere tetrapteron (Bonaduce, Ciampo \& Masoli);

Colalongo \& Pasini: 120, pl. 34 fig. 2.
1985 Tuberculocythere tetrapteron (Bonaduce, Ciampo \& Masoli); Moncharmont-Zei et al.: 28, pl. 1, fig. 1.

1994 Parahemingwayella tetrapteron (Bonaduce, Ciampo \& Masoli); Malz \& Jellinek: 28, pl. 7, figs 37-40.

2000 Parahemingwayella tetrapteron (Bonaduce, Ciampo \& Masoli); Aiello et al.: 94, pl. 3, fig. 9.

2001 Eucytherura sp. Didié \& Bauch (as erratum of Didié \& Bauch, 2000): 103, pl. 1, fig. 11.

2005 Parahemingwayella tetrapteron (Bonaduce, Ciampo \& Masoli); Guernet: 108.

Remarks. Eucytherura tetrapteron (Bonaduce, Ciampo \& Masoli, 1976 ) is very similar to E. downingae (Coles \& Whatley, 1989), but the latter has more strongly developed primary and secondary reticulation and bears two spines on the anterodorsal margin (the former bears a continuous frill). Although these differences are subtle and may be a result of intraspecific variation in calcification, we consider these two as separate species at least for now. E. tetrapteron is known from the Mediterranean and the eastern North Atlantic.

Genus Kangarina Coryell \& Fields, 1937

Type species. Kangarina quellita Coryell \& Fields, 1937 Kangarina abyssicola (Müller, 1894)

(Fig. 11D-E)

1894 Cytheropteron abyssicolum Müller: 302, pl. 20, figs 5, 11; pl. 21, figs 6-9.

1952 Cytheropteron (Kangarina) abyssicolum Müller; Ruggieri: 77, pl. 6, fig. 9 .

1953 Kangarina abyssicola coarctata Ruggieri: 53, figs 16, 16 a. 1953 Kangarina abyssicola (Müller); Ruggieri: 53, figs 15, 15a.

1972 Kangarina septentrionalis Neale: 33, pl. 1, figs 1-8.

1976 Kangarina abyssicola (Müller); Bonaduce et al.: 84, pl. 17, fig. 16.

1980 Kangarina abyssicola (Müller); Colalongo \& Pasini: 58, pl. 22 , fig. 2 .

1988 Kangarina abyssicola (Müller); Guernet \& Fourcade: 145, pl. 4, fig. 12 .

non 1993 Kangarina abyssicola (Müller); Witte: 43, pl. 9, figs $25-26$.

1996 Kangarina abyssicola (Müller); Coles et al.: 135, pl. 2, figs $10-11$.

2004 Kangarina? abyssicola (Müller); Aiello \& Szczechura: 53, pl. 8, fig. 15.

2005 Kangarina abyssicola (Müller); Guernet: 103.

2005 Kangarina coarctata Ruggieri; Guernet: 103.

$2009 b$ Kangarina cf. abyssicola (Müller); Yasuhara et al.: 914, p. 14, fig. 13 .

2010 Kangarina abyssicola (Müller); Aiello \& Barra: 412.

Remarks. We consider Kangarina coarctata Ruggieri, 1953 and K. septentrionalis Neale, 1972 as junior synonyms of K. abyssicola (Müller, 1894). K. abyssicola is known from the Mediterranean and the eastern and western North Atlantic.

Genus Pedicythere Eagar, 1965

Type species. Pedicythere tessae Eagar, 1965

Remarks. We found four Pedicythere species in ODP 982A, all of which also occur in the western North Atlantic (Yasuhara et al., 2009b). 


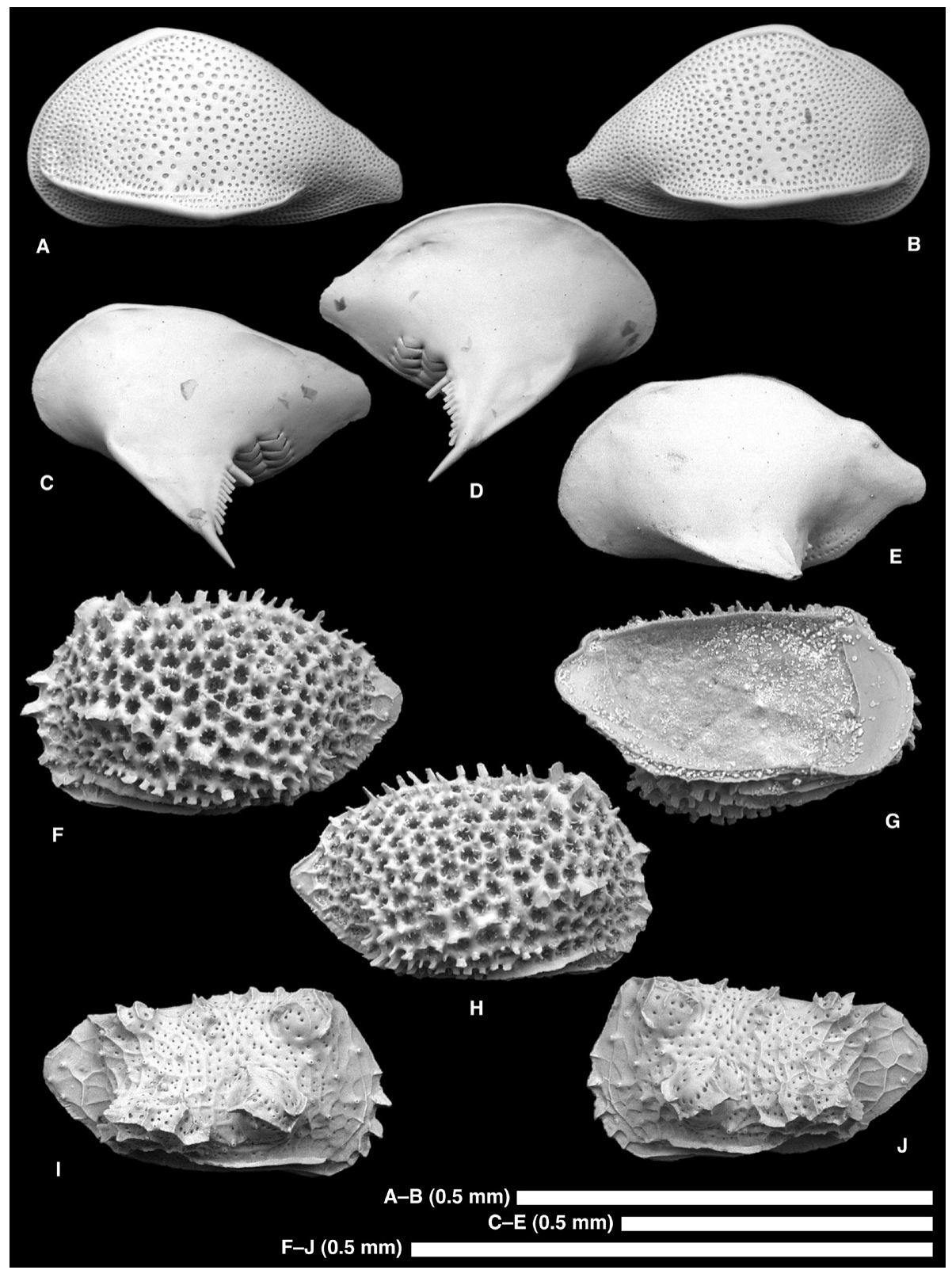

Fig. 10. SEM images of Cytheropteron and Eucytherura species. A-B, Cytheropteron perlaria Hao, 1988: A, USNM 603706 (ODP982033); adult LV from 1/2/17-19; B, USNM 603707 (ODP982034); adult RV from 1/2/17-19. C-D, Cytheropteron pherozigzag Whatley, Ayress \& Downing, 1986: C, USNM 603708 (ODP982032); adult LV from 1/1/142-144; D, USNM 603709 (ODP982031); adult RV from 1/2/17-19. E, Cytheropteron pseudoalatum Colalongo \& Pasini, 1980, USNM 603710 (ODP982094); adult LV from 1/2/127-129. F-H, Eucytherura calabra (Colalongo \& Pasini, 1980): F, USNM 603711 (ODP982100); adult LV from 1/1/70-72; G, USNM 603712 (ODP982101); adult LV from 1/1/90-92; H, USNM 603713 (ODP982102); adult RV from 1/1/107-109. I-J, Eucytherura multituberculata Ayress, Whatley, Downing \& Millson, 1995: I, USNM 603714 (ODP982004); adult RV from 1/3/112-114; J, USNM 603715 (ODP982005); adult LV from 1/3/92-94. A-F, H-J, lateral views; G, internal view. All specimens from late Quaternary section of ODP Hole 982A, Rockall Plateau, eastern North Atlantic. Scale bars represent $0.5 \mathrm{~mm}$.

Pedicythere atroposopetasi Yasuhara, Okahashi \& Cronin, 2009 (Figs 11F-I, 12A-D)

?2000 Pedicythere sp. B Guernet \& Bellier: 270, pl. 5, fig. 3. 2009 Pedicythere atroposopetasi Yasuhara, Okahashi \& Cronin: 914, pl. 15, figs $1-13$.

Pedicythere kennettopetasi Yasuhara, Okahashi \& Cronin, 2009 (Fig. 13E-F)
?2000 (part) Pedicythere sp. A Guernet \& Bellier: 270, pl. 5, fig. 2 (non fig. 1).

2009 Pedicythere kennettopetasi Yasuhara, Okahashi \& Cronin: 916, pl. 16, figs $1-10$.

Pedicythere klothopetasi Yasuhara, Okahashi \& Cronin, 2009

(Figs 13G-J, 14A-E)

2009 Pedicythere klothopetasi Yasuhara, Okahashi \& Cronin: 916, pl. 15, figs 14-21. 


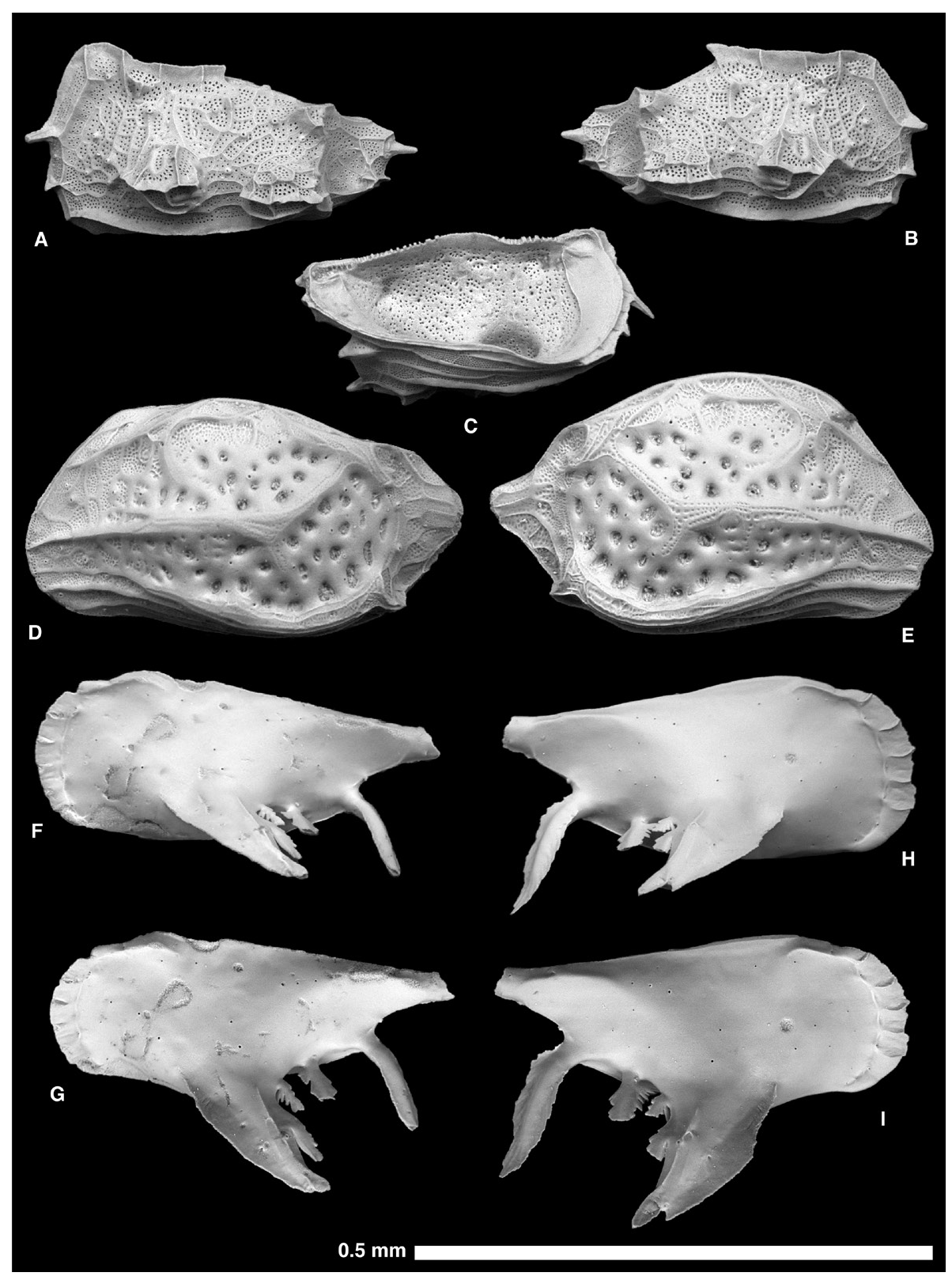

Fig. 11. SEM images of Eucytherura, Kangarina and Pedicythere species. A-C, Eucytherura tetrapteron (Bonaduce, Ciampo \& Masoli, 1976): A, USNM 603716 (ODP982001); adult LV from 1/3/112-114; B, USNM 603717 (ODP982002); adult RV from 1/3/112-114; C, USNM 603718 (ODP982003); adult LV from 1/3/112-114. D-E, Kangarina abyssicola (Müller, 1894): D, USNM 603719 (ODP982104); adult LV from 1/1/137-139; E, USNM 603720 (ODP982103); adult RV from 1/1/70-72. F-I, Pedicythere atroposopetasi Yasuhara, Okahashi \& Cronin, 2009: F-G, USNM 603721 (ODP982136); adult LV from 1/3/82-84; H-I, USNM 603722 (ODP982137); adult RV from 1/3/82-84. A-B, D-F, H, lateral views; C, internal view; G, I, oblique views. All specimens from late Quaternary section of ODP Hole 982A, Rockall Plateau, eastern North Atlantic. Scale bar represents $0.5 \mathrm{~mm}$.

Pedicythere lachesisopetasi Yasuhara, Okahashi \& Cronin, 2009 (Figs 12E-J, 13A-D)

1983 Pedicythere sp. A Cronin: 110, pl. 4H.

2008 Pedicythere sp. Bergue \& Coimbra: 130, pl. 6, fig. 13.

2009 Pedicythere lachesisopetasi Yasuhara, Okahashi \& Cronin:

918, pl. 16, figs 11-21.

Family Eucytheridae Puri, 1954

Genus Eucythere Brady, 1868
Type species. Cythere declivis Norman, 1867 (designated by Brady \& Norman, 1889; see Horne \& Whittaker (1985) for details and lectotype).

Eucythere triangula Whatley \& Coles, 1987

(Fig. 14F)

1987 Eucythere triangula Whatley \& Coles: 74, pl. 4, figs 16-18. 2000 Eucythere triangula Whatley \& Coles; Didié \& Bauch: 114, pl. 3, fig. 21. 


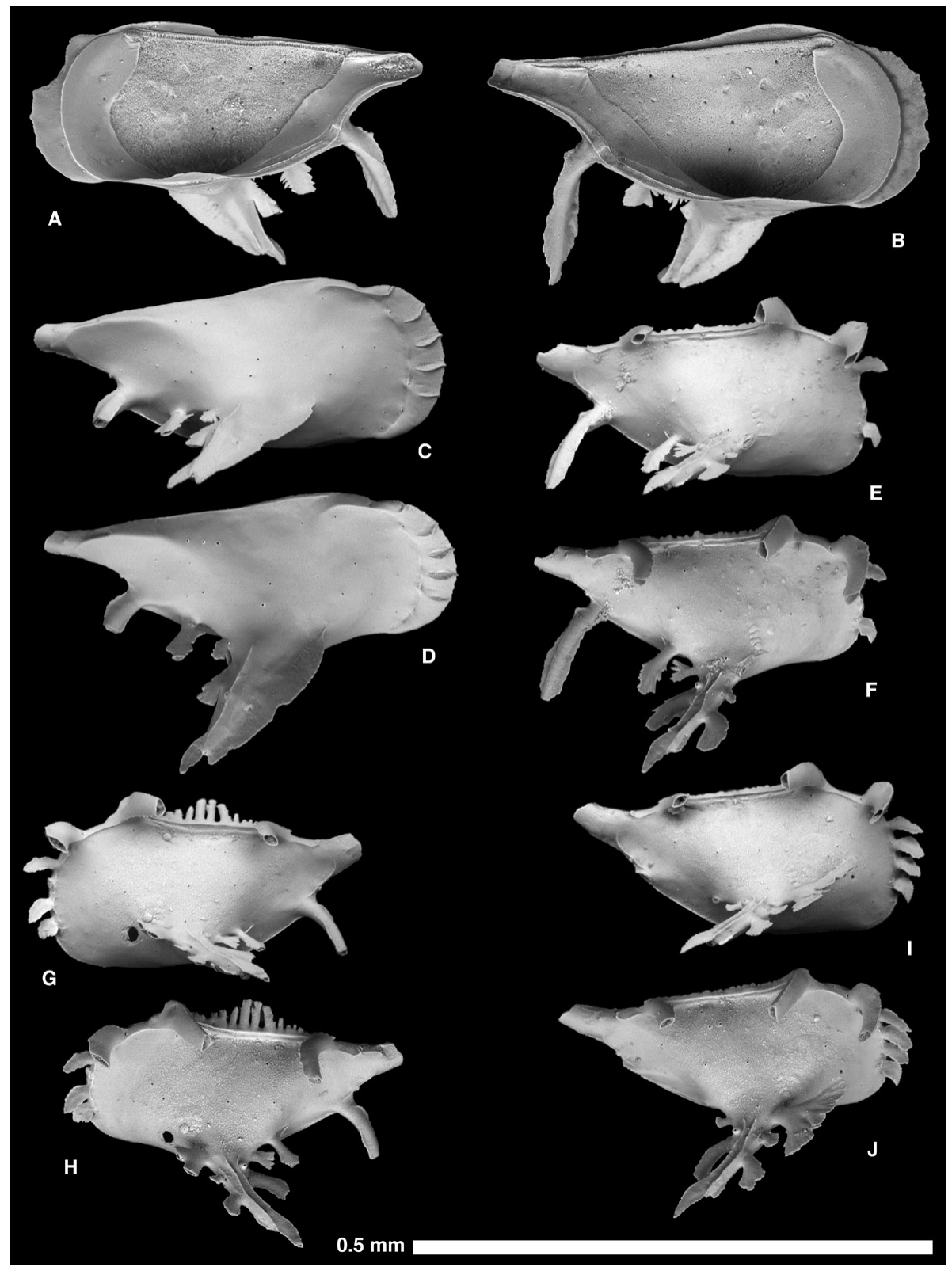

Fig. 12. SEM images of Pedicythere species. A-D, Pedicythere atroposopetasi Yasuhara, Okahashi \& Cronin, 2009: A, USNM 603723 (ODP982138); adult RV from 1/1/70-72; B, USNM 603724 (ODP982139); adult LV from 1/3/92-94; C-D, USNM 603725 (ODP982146); adult RV from 1/1/142144. E-J, Pedicythere lachesisopetasi Yasuhara, Okahashi \& Cronin, 2009: E-F, USNM 603726 (ODP982140); adult RV from 1/1/97-99; G-H, USNM 603727 (ODP982141); adult LV from 1/3/122-124; I-J, USNM 603728 (ODP982142); adult RV from 1/3/122-124. C, E, G, I, lateral views; A-B, internal views; D, F, H, J, oblique views. All specimens from late Quaternary section of ODP Hole 982A, Rockall Plateau, eastern North Atlantic. Scale bar represents $0.5 \mathrm{~mm}$.

2009 Eucythere triangula Whatley \& Coles; Alvarez Zarikian: 4, pl. P6, fig. 4.

2009 bucythere triangula Whatley \& Coles; Yasuhara et al.: 920, pl. 17, figs $2-7$.

Remarks. This species is known both from the eastern and western North Atlantic.

Family Krithidae Mandelstam, 1958 (in Bubikyan, 1958) Genus Krithe Brady, Crosskey \& Robertson, 1874

Type species. Ilyobates praetexta Sars, 1866
Remarks. Krithe is one of the most abundant genera in this core, representing on average $20 \%$ of the total fauna. We followed the taxonomy of Coles et al. (1994). Krithe in this core is mainly composed of $K$. dolichodeira van den Bold, 1946, K. ayressi Coles et al., 1994 and K. minima Coles et al., 1994.

Family Leptocytheridae Hanai, 1957 Genus Cluthia Neale, 1973

Type species. Cythere cluthae Brady, Crosskey \& Robertson, 1874 


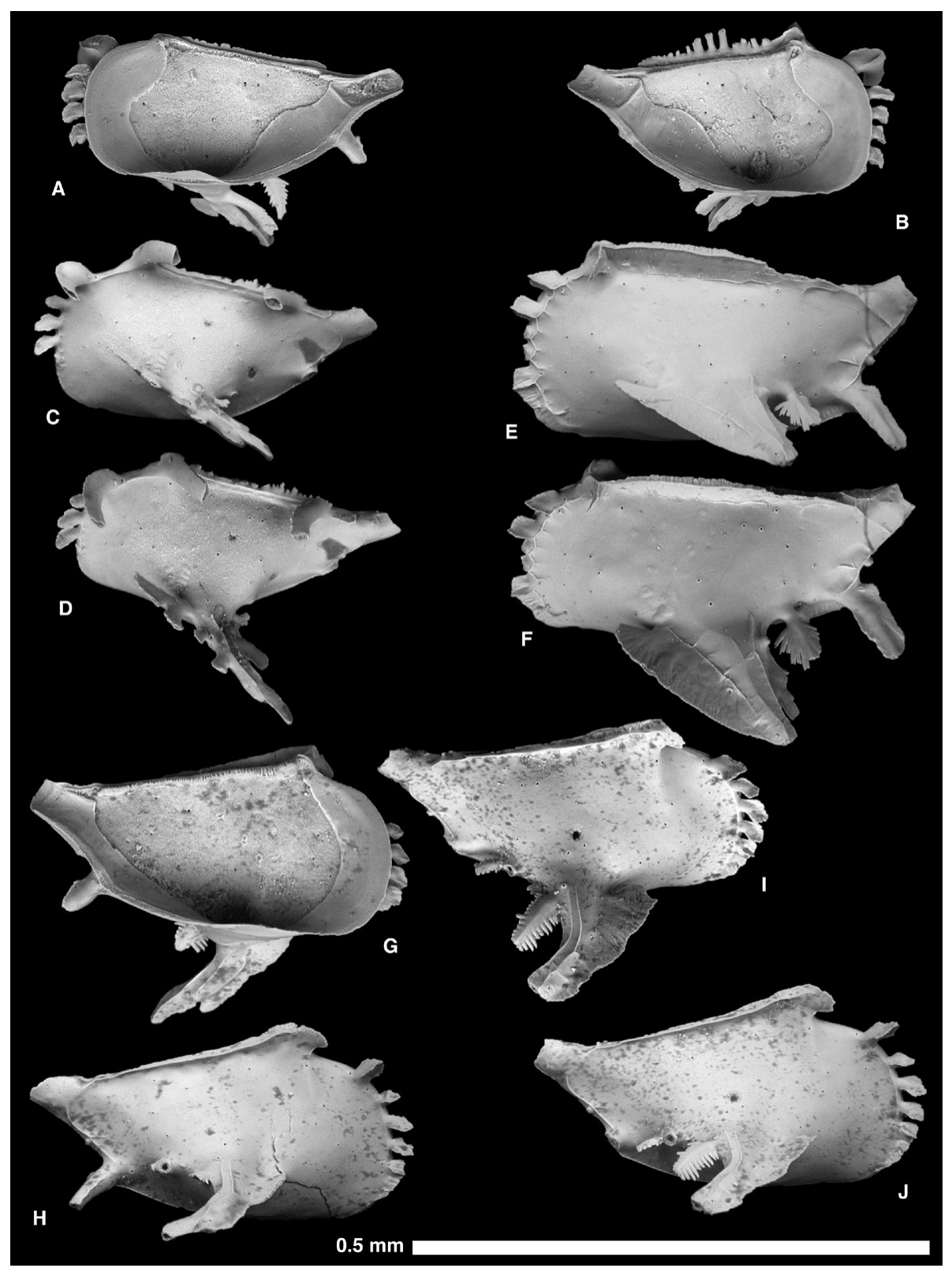

Fig. 13. SEM images of Pedicythere species. A-D, Pedicythere lachesisopetasi Yasuhara, Okahashi \& Cronin, 2009: A, USNM 603729 (ODP982144); adult RV from 1/3/122-124; B, USNM 603730 (ODP982145); adult LV from 1/1/60-62; C-D, USNM 603731 (ODP982143); adult LV from 1/3/122-124. E-F, Pedicythere kennettopetasi Yasuhara, Okahashi \& Cronin, 2009, USNM 603732 (ODP982147); adult LV from 1/3/7274. G-J, Pedicythere klothopetasi Yasuhara, Okahashi \& Cronin, 2009: G, USNM 603733 (ODP982148); adult LV from 1/1/80-82; H, USNM 603734 (ODP982149pedi); adult RV from 1/1/80-82; I-J, USNM 603735 (ODP982150); adult RV from 1/1/80-82. C, E, H, J, lateral views; A-B, G, internal views; D, F, I, oblique views. All specimens from late Quaternary section of ODP Hole 982A, Rockall Plateau, eastern North Atlantic. Scale bar represents $0.5 \mathrm{~mm}$.

Cluthia sp.

(Fig. 14G-J)

1998 Nannocythere sp. Whatley, Eynon \& Moguilevsky: 23, pl. 3, figs 9-10.

?2000 Nannocythere sp. Didié \& Bauch: 111, pl. 4, fig. 25.

Remarks. This species is formally described as new in Yasuhara et al. (in press $b$ ).
Family Loxoconchidae Sars, 1926

Genus Loxoconchidea Bonaduce, Ciampo \& Masoli, 1976 Type species. Loxoconchidea minima Bonaduce, Ciampo \& Masoli, 1976

Loxoconchidea minima Bonaduce, Ciampo \& Masoli, 1976 (Fig. 15A)

1976 Loxoconchidea minima Bonaduce, Ciampo \& Masoli: 112, pl. 59, figs $1-7$, text-fig. 43 . 


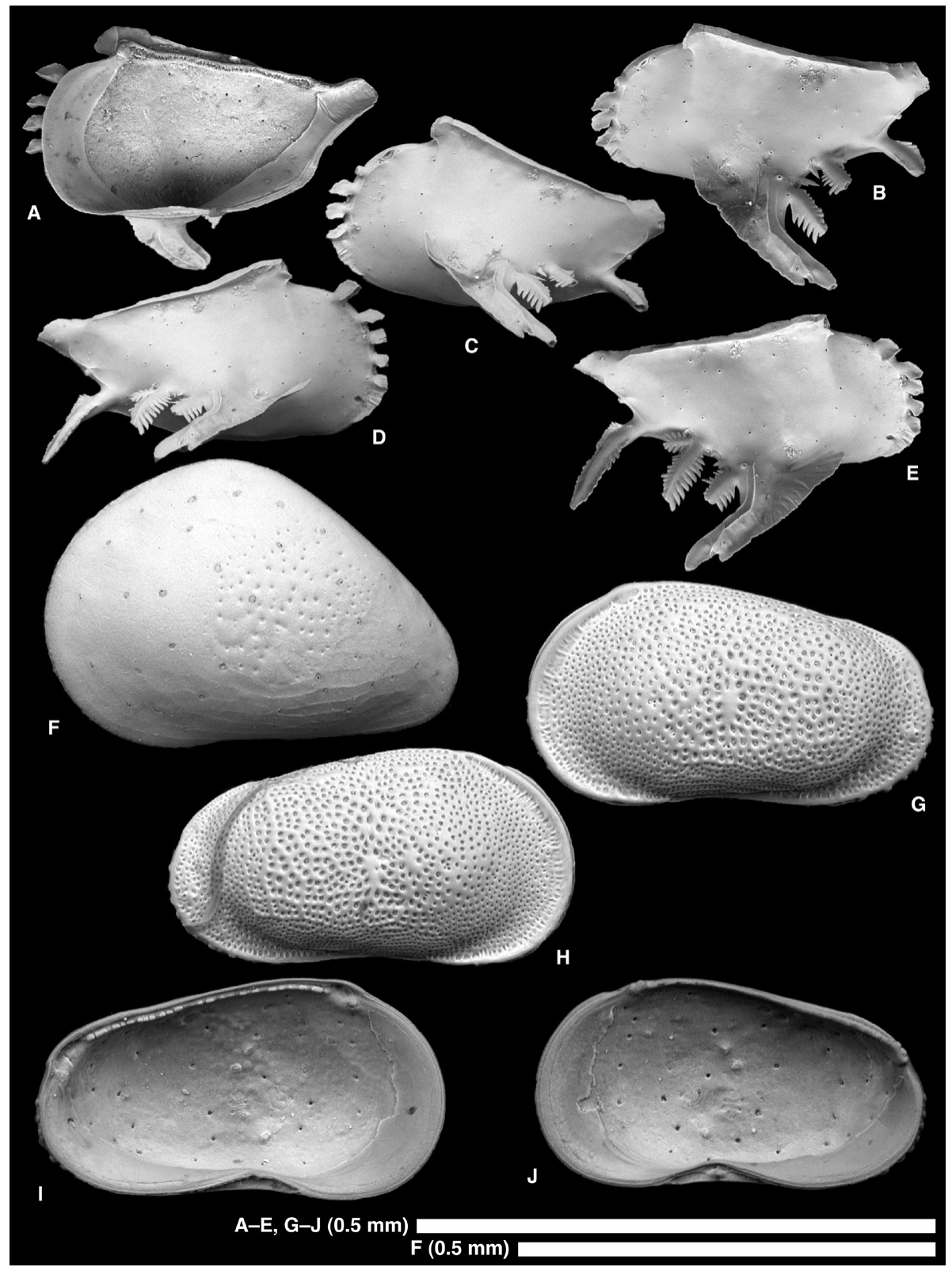

Fig. 14. SEM images of Pedicythere, Eucythere and Cluthia species. A-E, Pedicythere klothopetasi Yasuhara, Okahashi \& Cronin, 2009: A, USNM 603736 (ODP982151); adult RV from 1/1/80-82; B-C, USNM 603737 (ODP982152); adult LV from 1/1/70-72; D-E, USNM 603738 (ODP982153); adult RV from 1/1/70-72. F, Eucythere triangula Whatley \& Coles, 1987, USNM 603739 (ODP982099); adult LV from 1/2/117-119. G-J, Cluthia sp.: G, USNM 603740 (ODP982127-2); adult LV from 1/3/12-14; H, USNM 603741 (ODP982128); adult RV from 1/3/92-94; I, USNM 603742 (ODP982129-1); adult LV from 1/3/92-94; J, USNM 603743 (ODP982130); adult RV from 1/3/92-94. C-D, F-H, lateral views; A, I-J, internal views; B, E, oblique views. All specimens from late Quaternary section of ODP Hole 982A, Rockall Plateau, eastern North Atlantic. Scale bars represent $0.5 \mathrm{~mm}$.

2000 Loxoconchidea minima Bonaduce, Ciampo \& Masoli; Aiello et al.: 97, pl. 3, fog. 10 .

2004 Loxoconchidea minima Bonaduce, Ciampo \& Masoli; Aiello \& Szczechura: 35, pl. 7, figs 1-3.

2006 Loxoconchidea minima Bonaduce, Ciampo \& Masoli; Bergue et al:: 206, fig. 6E.

2008 Loxoconchidea minima Bonaduce, Ciampo \& Masoli; Bergue \& Coimbra: 115, pl. 1, fig. 16.

2009 b Loxoconchidea minima Bonaduce, Ciampo \& Masoli; Yasuhara et al.: 920, pl. 17, figs 8-11.
Remarks. A comprehensive synonymy list can be found in Aiello \& Szczechura (2004) and Yasuhara et al. (2009b). This species is known from the Atlantic and Mediterranean regions.

Family Paradoxostomatidae Brady \& Norman, 1889 Genus Paracytherois Müller, 1894

Type species. Paracytherois striata Müller, 1894 [designated by Howe, 1955 (he considered this species a junior synonym of Paradoxostoma flexuosum Brady (1868) (sic: correctly, Bythocythere? flexuosa Brady, (1867)); see Ellis \& Messina Catalogue]. 


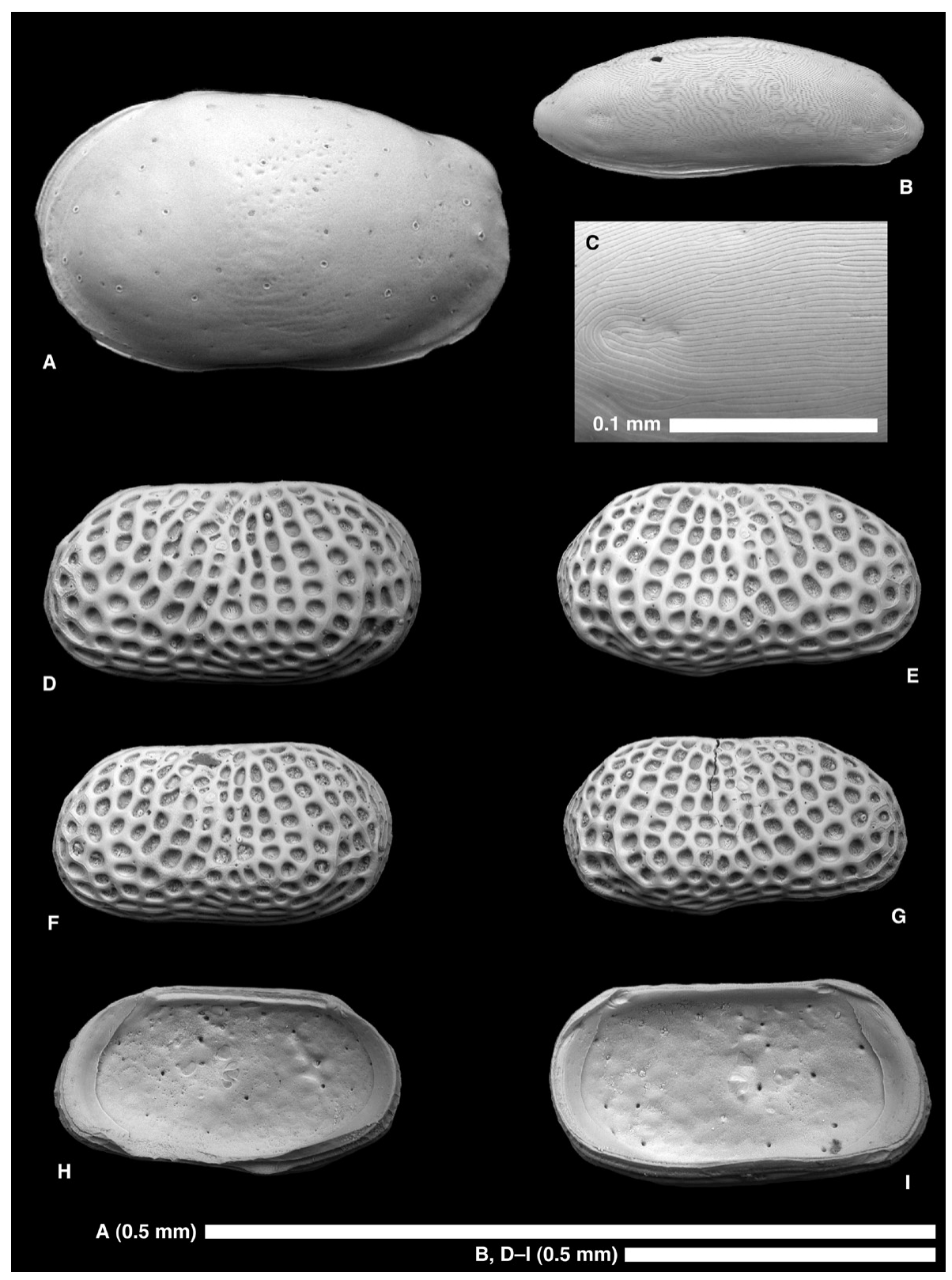

Fig. 15. SEM images of Loxoconchidea, Paracytherois, and Arcacythere species. A, Loxoconchidea minima Bonaduce, Ciampo \& Masoli, 1976, USNM 603744 (ODP982123); adult LV from 1/3/12-14. B-C, Paracytherois bondi Yasuhara, Okahashi \& Cronin, 2009: USNM 603745 (ODP982135); adult RV from 1/1/80-82. D-I, Arcacythere enigmatica (Whatley, Frame \& Whittaker, 1978): D, USNM 603746 (ODP982169rock); adult LV from 1/2/7779; E, USNM 603747 (ODP982170rock); adult RV from 1/2/77-79; F, USNM 603748 (ODP982171rock); adult LV from 1/2/77-79; G, USNM 603749 (ODP982172rock); adult RV from 1/2/77-79; H, USNM 603750 (ODP982173rock); adult RV from 1/2/67-69; I, USNM 603751 (ODP982174rock); adult LV from 1/3/92-94. A-G, lateral views; H-I, internal views. All specimens from late Quaternary section of ODP Hole 982A, Rockall Plateau, eastern North Atlantic. Scale bars represent $0.5 \mathrm{~mm}$ for A-B, D-I and $0.1 \mathrm{~mm}$ for C.

Paracytherois bondi Yasuhara, Okahashi \& Cronin, 2009

(Fig. 15B-C)

2009 b Paracytherois bondi Yasuhara, Okahashi \& Cronin: 924, pl. 19, figs 5-10, 15 (?12).

Remarks. This species was known only from the western North Atlantic, but this record confirms its presence in the eastern North Atlantic.
Family Rockalliidae Whatley, Uffenorde, Harlow, Downing \& Kesler, 1982

Genus Arcacythere Hornibrook, 1952

Type species. Arcacythere chapmani Hornibrook, 1952

Remarks. We agree with Ayress (1991) in considering Rockallia Whatley, Frame \& Whittaker, 1978 as a junior synonym of Arcacythere Hornibrook, 1952. See Yasuhara \& Okahashi (in press) for detailed discussion. 


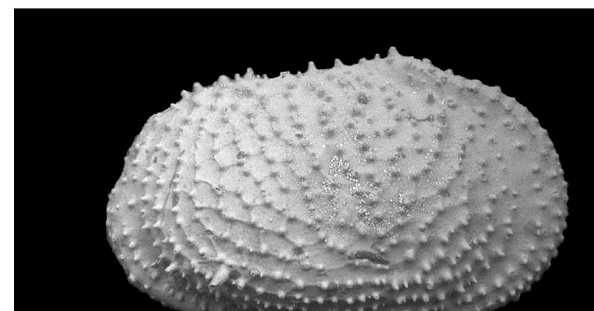

A
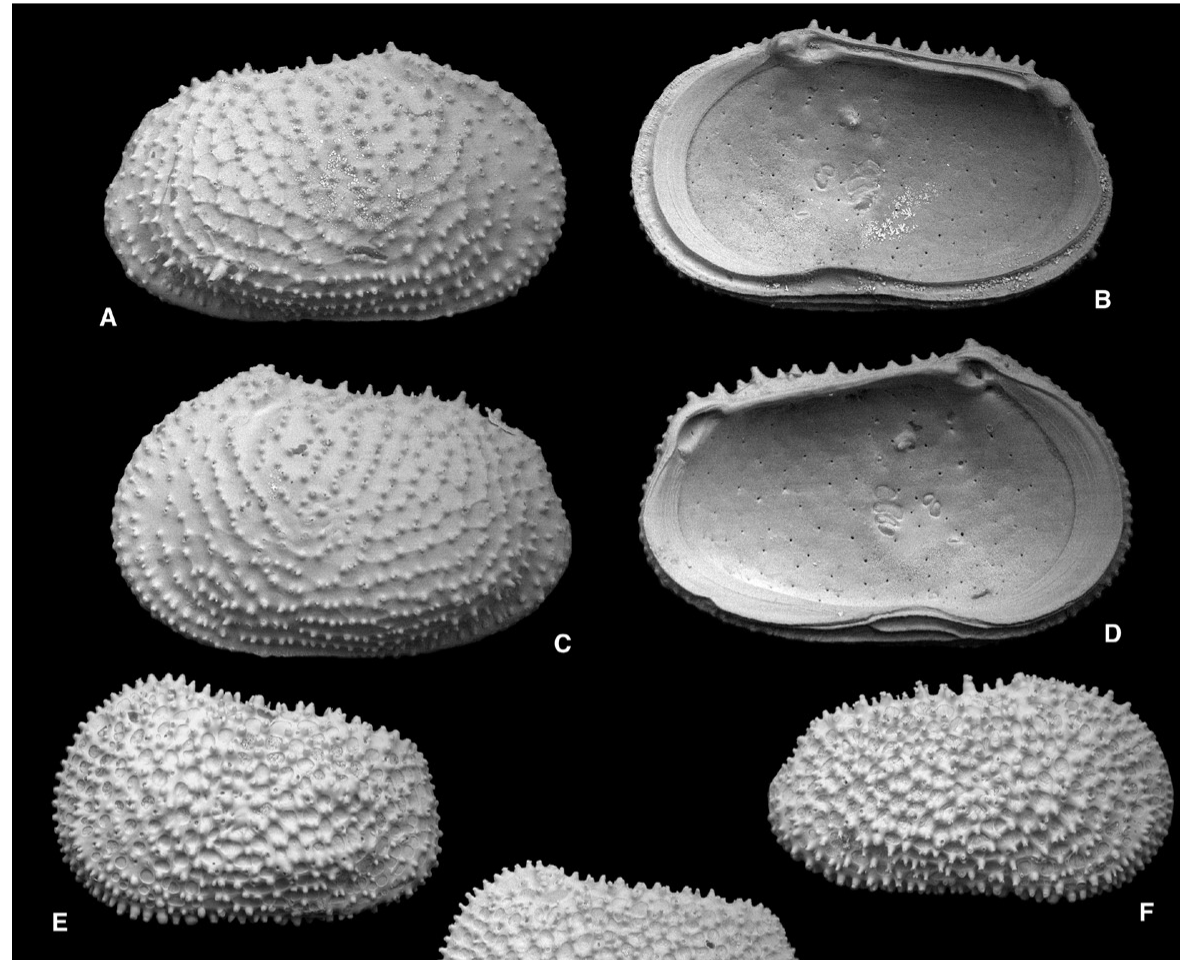

E
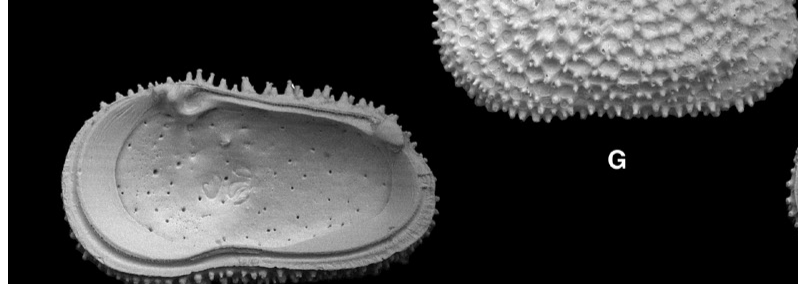

H

G
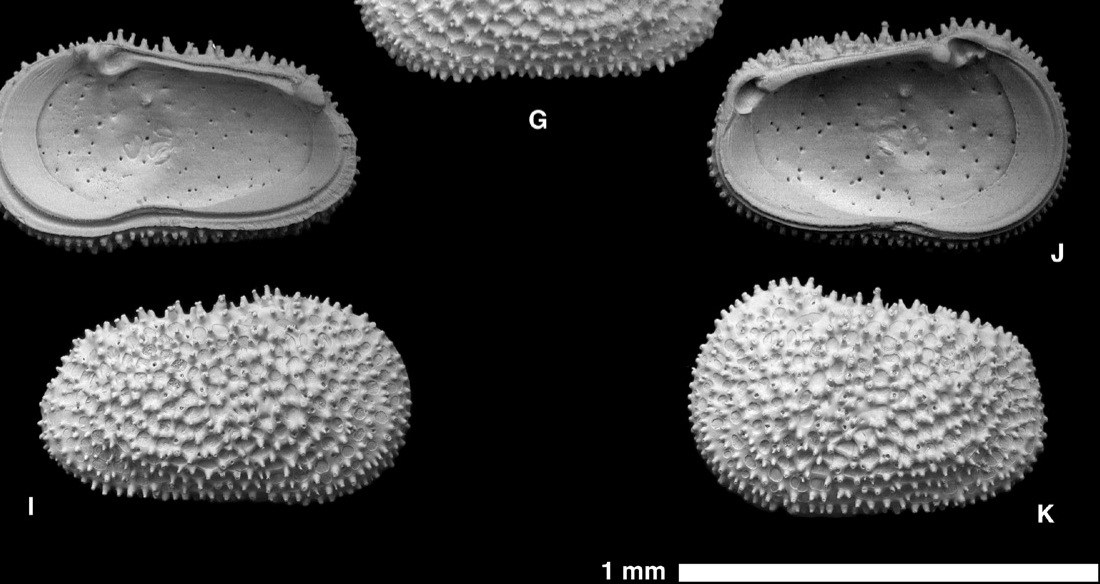

Fig. 16. SEM images of Echinocythereis and Henryhowella species. A-D, Echinocythereis echinata (Sars, 1866): A, USNM 603752 (ODP982027); adult RV from 1/1/142-144; B, USNM 603753 (ODP982028); adult RV from 1/1/142-144; C, USNM 603754 (ODP982029); adult LV from 1/3/72-74; D, USNM 603755 (ODP982030); adult LV from 1/3/72-74. E-K, Henryhowella asperrima (Reuss, 1850): E, USNM 603756 (ODP982022); adult LV from 1/1/137-139; F, USNM 603757 (ODP982023); adult RV from 1/1/137-139; G, USNM 603758 (ODP982026); adult LV from 1/1/0-2; H-I, USNM 603759 (ODP982024); adult RV from 1/1/142-144; J-K, USNM 603760 (ODP982025); adult LV from 1/1/107-109. A, C, E-G, I, K, lateral views; B, D, H, J, internal views. All specimens from late Quaternary section of ODP Hole 982A, Rockall Plateau, eastern North Atlantic. Scale bar represents $1 \mathrm{~mm}$.

Arcacythere enigmatica (Whatley, Frame \& Whittaker, 1978)

(Fig. 15D-I)

1978 Rockallia enigmatica Whatley, Frame \& Whittaker: 137, pls 5-138, 5-140, 5-142, 5-144; text-fig. 1.

1979 Indet. Gen. 3 Ducasse \& Peypouquet: pl. 5, fig. 9.

1982 Rockallia enigmatica Whatley, Frame \& Whittaker; Whatley et al.: 3, pl. 1, figs 1, 4 .

1987 Rockallia enigmatica Whatley, Frame \& Whittaker; Whatley \& Coles: 80, pl. 2, figs 3-4.
1987 Rockallia sp. Whatley \& Coles: 89, pl. 2, fig. 5.

1988 Rockallia enigmatica Whatley, Frame \& Whittaker; Ruan \& Hao: 377, pl. 70, figs 2-4.

1988 Rockallia inceptiocelata Whatley, Uffenorde, Harlow, Downing \& Kesler; Ruan \& Hao: 377, pl. 70, figs 5-7.

1990 Rockallia enigmatica Whatley, Frame \& Whittaker; Malz: 143 , fig. 4.2.

2000 Rockallia enigmatica Whatley, Frame \& Whittaker; Didié \& Bauch: 116, pl. 3, figs 13-14. 
2003 Rockallia enigmatica Whatley, Frame \& Whittaker; Cronin \& Dwyer: 263, pl. 2, fig. n.

2005 Rockallia enigmatica Whatley, Frame \& Whittaker; Mazzini: 86 , figs 50P, 51B.

2007 Rockallia enigmatica Whatley, Frame \& Whittaker; Hou \& Gou: 509, pl. 198, figs 1-4.

2007 Rockallia inceptiocelata Whatley, Uffenorde, Harlow, Downing \& Kesler; Hou \& Gou: 509, pl. 198, figs 5, 8 (?6-7).

2009 Rockallia enigmatica Whatley, Frame \& Whittaker; Alvarez Zarikian: 5, pl. P9, fig. 5.

Remarks. This species is known from the eastern North Atlantic and northwestern Pacific oceans.

Family Trachyleberididae Sylvester-Bradley, 1948

Remarks. Ambocythere, Buntonia and Pennyella occur in this core, but we will discuss these genera elsewhere.

Genus Echinocythereis Puri, 1954

Type species. Cythere margaritifera Brady, 1870 [=Cythereis garretti Howe \& McGuirt, 1935 (in Howe \& graduate students, 1935); see Hazel (1967)].

\section{Echinocythereis echinata (Sars, 1866)}

(Fig. 16A-D)

1866 Cythereis echinata Sars: 44.

1880 Cythere irpex Brady: 107, pl. 17, fig. 2a-d.

?1967 Echinocythereis echinata (Sars); Hazel: 37, pl. 6, figs 10-11.

1976 Cythere irpex Brady; Puri \& Hulings: 278, pl. 11, figs 1-9.

1990 Echinocythereis whatleyi Dingle, Lord \& Boomer: 303, figs 35B-F, 36E-G, I-J.

2000 Echinocythereis echinata (Sars); Barra \& Bonaduce: 214, pl.

1 , figs 1-10; text-fig. 1.

2004 Echinocythereis echinata (Sars); Ayress et al.: 35, pl. 3, fig. 9.

2009 Echinocythereis echinata (Sars); Alvarez Zarikian: 6, pl. P9, figs 3-4.

2009 bchinocythereis echinata (Sars); Yasuhara et al.: 926, pl. 21, figs 6-9.

Remarks. A comprehensive synonymy and detailed discussion are found in Yasuhara et al. $(2009 b)$ and references therein. This species is known from the Atlantic and Southern Oceans.

Genus Henryhowella Puri, 1957

Type species. Cythere evax Ulrich \& Bassler, 1904 Henryhowella asperrima (Reuss, 1850)

(Fig. 16E-K)

1850 Cypridina asperrima Reuss: 74, pl. 10, fig. 5a-b.

2005 Henryhowella asperrima (Reuss); Mazzini: 50, figs 26A-I, 27B.

2009 Henryhowella dasyderma (Brady); Alvarez Zarikian: 6, pl. 9, figs 6-8.

$2009 b$ Henryhowella cf. asperrima (Reuss); Yasuhara et al.: 926, pl. 20, fig. 7; pl. 21, figs $1-4$.

2010 Henryhowella asperrima (Reuss); Bergue \& Govindan: 751, fig. 3.14.

2011 Henryhowella asperrima (Reuss); Pirkenseer \& Berger: 54, pl. 7 , figs $6 a-6 c, 7 a-7 c ;$ pl. 8 , figs $1 a-1 c, 2 a-2 c, 3 a-3 c$.

Remarks. To be discussed in detail elsewhere.

\section{DISCUSSION}

ODP 982A late Quaternary ostracod assemblages show a strong affinity to the western North Atlantic and Mediterranean bathyal faunas and, to a lesser extent, to the western North Pacific bathyal fauna. There are many common or closely related species among these regions, as shown in the Systematic Palaeontology section above. The strong affinity among the western North Atlantic, the eastern North Atlantic and the Mediterranean faunas is understandable, given their proximity and bathyal-depth connections (e.g. Strait of Gibraltar and Greenland-Iceland-Faeroe Ridge). However, an affinity between the North Atlantic and the North Pacific is more difficult to explain. Two possibilities are proposed: (1) many bathyal ostracod species are cosmopolitan; or (2) they were able to migrate through the Bering Strait despite its present-day shallow depth $(<50 \mathrm{~m})$. We will need further modern and palaeo-biogeographical studies to evaluate these hypotheses.

\section{ACKNOWLEDGEMENTS}

We thank D. A. Hodell for kindly loaning ODP 982A samples; L. M. Y. Wong and C. Sanford for continuous support; S. Whittaker for help with SEM imaging; G. Hunt for help in depositing type and figured specimens; C. Alvarez Zarikian and an anonymous reviewer for valuable comments. Samples used for this research were provided by the Integrated Ocean Drilling Program (IODP). This work was supported by the Seed Funding Programme for Basic Research of the University of Hong Kong (project codes: 201105159002; 201210159043), Hung Hing Ying Physical Sciences Research Fund 2012-13, Smithsonian Postdoctoral Fellowship, and Smithsonian Marine Science Network Postdoctoral Fellowship (to M.Y.).

\section{Manuscript received 8 September 2013}

Manuscript accepted 15 January 2014

Scientific Editing by Alan Lord.

\section{REFERENCES}

Aiello, G. \& Barra, D. 2010. Crustacea Ostracoda. Biologia Marina Mediterranea, 17: 401-419.

Aiello, G. \& Szczechura, J. 2004. Middle Miocene ostracods of the Fore-Carpathian Depression (Central Paratethys, southwestern Poland). Bollettino della Societá Paleontologica Italiana, 43: 11-70.

Aiello, G., Barra, D. \& Bonaduce, G. 1996a. The genus Cytheropteron Sars, 1866 (Crustacea: ostracoda) in the Pliocene-Early Pleistocene of the Mount San Nicola Section (Gela, Sicily). Micropaleontology, 42: $167-178$.

Aiello, G., Barra, D., Bonaduce, G. \& Russo, A. 1996b. The genus Cytherella Jones, 1849 (Ostracoda) in the Italian Tortonian-recent. Revue de Micropaléontologie, 39: 171-190.

Aiello, G., Barra, D. \& Bonaduce, G. 2000. Systematics and biostratigraphy of the Ostracoda of the Plio-Pleistocene Monte S. Nicola section (Gela, Sicily). Bollettino della Societá Paleontologica Italiana, 39: 83-112.

Alexander, C.I. 1936. Ostracoda of the Genera Eucythere, Cytherura, Eucytherura and Loxoconcha from the Cretaceous of Texas. Journal of Paleontology, 10: 689-694.

Alvarez Zarikian, C.A. 2009. Data report: Late Quaternary ostracodes at IODP Site U1314 (North Atlantic Ocean). Proceedings of the Integrated Ocean Drilling Program, 303/306: 1-22.

Athersuch, J., Horne, D.J. \& Whittaker, J.E. 1989. Marine and Brackish Water Ostracods. In Synopses of the British Fauna (New Series) No. 43. The Linnean Society of London and the Estuarine and Brackish-Water Science Association, London, 343pp. 
Ayress, M.A. 1991. On Arcacythere Hornibrook, 1952 (Cytheracea, Ostracoda, Crustacea), a senior synonym of Rockallia Whatley, Frame \& Whittaker, 1978. Journal of Micropalaeontology, 10: 223-226.

Ayress, M.A., Whatley, R.C., Downing, S.E. \& Millson, K.J. 1995. Cainozoic and Recent deep sea Cytherurid Ostracoda from the south western Pacific and eastern Indian Oceans, part I: Cytherurinae. Records of the Australian Museum, 47: 203-223.

Ayress, M.A., De Deckker, P. \& Coles, G.P. 2004. A taxonomic and distributional survey of marine benthonic Ostracoda off Kerguelen and Heard Islands, South Indian Ocean. Journal of Micropalaeontology, 23: 15-38.

Baird, W. 1850. The Natural History of the British Entomostraca. Ray Society, London, 364pp.

Barra, D. \& Bonaduce, G. 2000. Some species of Echinocythereis Puri, 1954 (Crustacea, Ostracoda) from the Torronian and to Recent. Revista Española de Micropaleontología, 32: 213-224.

Barra, D., Aiello, G. \& Bonaduce, G. 1996. The genus Argilloecia Sars, 1866 (Crustacea: Ostracoda) in the Pliocene-Early Pleistocene of the M. San Nicola Section (Gela, Sicily). Proceedings of the 2nd European Ostracodologists Meeting, July 1993, Glasgow, UK, 129-134.

Bergue, C.T. \& Coimbra, J.C. 2008. Late Pleistocene and Holocene bathyal ostracodes from the Santos Basin, southeastern Brazil. Palaeontographica, A, 285: 101-144.

Bergue, C.T. \& Govindan, A. 2010. Eocene-Pliocene deep sea ostracodes from ODP site 744A, southern Indian Ocean. Anais da Academia Brasileira de Ciências, 82: 747-760.

Bergue, C.T., Costa, K.B., Dwyer, G. \& Moura, C.A.V. 2006. Bathyal ostracode diversity in the Santos Basin, Brazilian southeast margin: response to Late Quaternary climate changes. Revista Brasileira de Paleontologia, 9: 201-210.

Bonaduce, G., Ciampo, G. \& Masoli, M. 1976. Distribution of Ostracoda in the Adriatic Sea. Pubblicazioni della Stazione Zoologica di Napoli, 40: $1-154$

Bornemann, J.G. 1855. Die Mikroskopische Fauna des Septarienthones von Hermsdorfbei Berlin. Zeitschrift der Deutschen Geologischen Gesellschaft, 7: 307-371.

Brady, G.S. 1867. Report on the Ostracoda dredged amongst the Hebrides. Reports of the British Association for the Advancement of Science, 36: 208-211.

Brady, G.S. 1868. A monograph of the Recent British Ostracoda. Transactions of the Linnean Society of London, 26: 353-495.

Brady, G.S. 1870. Description of Ostracoda. In De Folin, L. \& Perier, L. (Eds), Les Fonds de la Mer. 1. Savy, Paris, 177-256.

Brady, G.S. 1880. Report on the Ostracoda dredged by H.M.S. Challenger, during the years 1873-1876. Report on the Scientific Results of the Exploring Voyage of H.M.S. Challenger. Zoology, 1: 1-184.

Brady, G.S. 1886. Les Ostracodes nouveaux des explorations du Travailleur et du Talisman. Les Fonds de la Mer, 4: 164-166, 194-200.

Brady, G.S. \& Norman, A.M. 1889. A monograph of the marine and fresh-water Ostracoda of the North Atlantic and of northwestern Europe. Section I: Podocopa. Scientific Transactions of the Royal Dublin Society, 4: 63-270.

Brady, G.S. \& Robertson, D. 1872. Contributions to the study of the Entomostraca. No. 6. On the distribution of the British Ostracoda. Annals and Magazine of Natural History, Series 4, 9, 48-70.

Brady, G.S., Crosskey, H.W. \& Robertson, D. 1874. A monograph of the post-Tertiary Entomostraca of Scotland including species from England and Ireland. Monograph of the Palaeontographical Society, 28: 1-232.

Breman, E. 1975. The distribution of ostracodes in the bottom sediments of the Adriatic Sea. PhD thesis, Vrije Universiteit te Amsterdam, 165pp.

Bubikyan, S.A. 1958. Ostracoda from Paleogene deposits of the Erevan Basin. Izvestiya Akademii Nuak Armyanskoy SSR, Seriya Geologicheskii i Geograficheskii Nauk, 11: 3-16.

Cabral, M.C. \& Loureiro, I.M. 2013. Overview of Recent and Holocene ostracods (Crustacea) from brackish and marine environments of Portugal. Journal of Micropalaeontology, 32: 135-159.
Ciampo, G. 1980. Ostracodi miocenici (Tortoniano-Messiniano) della regione di Ragusa (Sicilia). Bollettino della Societá Paleontologica Italiana, 19: 5-20.

Colalongo, M.L. \& Pasini, G. 1980. La ostracofauna plio-pleistocenica della Sezione Vrica in Calabria (con considerazioni sul limite Neogene/ Quaternario). Bollettino della Societá Paleontologica Italiana, 19: 44-126.

Coles, G.P. \& Whatley, R.C. 1989. New Palaeocene to Miocene genera and species of Ostracoda from DSDP sites in the North Atlantic. Revista Española de Micropaleontología, 21: 81-124.

Coles, G.P., Ayress, M.A. \& Whatley, R.C. 1990. A comparison of North Atlantic and Pacific deep-sea Ostracoda. In Whatley, R.C. \& Maybury, C. (Eds), Ostracoda and Global Events. Chapman \& Hall, London, 287-305.

Coles, G.P., Whatley, R.C. \& Moguilevsky, A. 1994. The ostracod genus Krithe from the Tertiary and Quaternary of the North Atlantic. Palaeontology, 37: 71-120.

Coles, G.P., Ainsworth, N.R., Whatley, R.C. \& Jones, R.W. 1996. Foraminifera and Ostracoda from Quaternary carbonate mounds associated with gas see page in the Porcupine Basin, offshore western Ireland. Revista Española de Micropaleontología, 28: 113-151.

Coryell, H.N. \& Fields, S. 1937. A Gatun ostracode fauna from Cativa, Panama. American Museum Novitates, 956: 1-18.

Coryell, H.N., Sample, C.H. \& Jennings, P.H. 1935. Bairdoppilata, a new genus of Ostracoda, with two new species. American Museum Novitates, 777: 1-5.

Cronin, T.M. 1983. Bathyal ostracodes from the Florida-Hatteras slope, the Straits of Florida, and the Blake Plateau. Marine Micropaleontology, 8: 89-119.

Cronin, T.M. \& Dwyer, G.S. 2003. Deep sea ostracodes and climatic change. The Paleontological Society Papers, 9: 247-263.

Cronin, T.M. \& Raymo, M.E. 1997. Orbital forcing of deep-sea benthic species diversity. Nature, 385: 624-627.

Cronin, T.M., DeMartino, D.M., Dwyer, G.S. \& Rodriguez-Lazaro, J. 1999. Deep-sea ostracode species diversity: response to late Quaternary climate change. Marine Micropaleontology, 37: 231-249.

Dall'Antonia, B. 2003. Miocene ostracods from the Tremiti Islands and Hyblean Plateau: biostratigraphy and description of new and poorly known species. Geobios, 36: 27-54.

Dana, J.D. 1853. Tribe III: Cyproidea = Ostracoda. Crustacea. United States Exploring Expedition during the Years 1838, 1839, 1840, 1841, 1842, under the Command of Charles Wilkes, U.S.N., with Atlas of 96 plates, 13: 1277-1304.

Didié, C. \& Bauch, H.A. 2000. Species composition and glacial-interglacial variations in the ostracode fauna of the northeast Atlantic during the past 200,000 years. Marine Micropaleontology, 40: 105-129.

Didié, C. \& Bauch, H.A. 2001. Erratum to 'Species composition and glacial-interglacial variations in the ostracode fauna of the northeast Atlantic during the past 200,000 years'. Marine Micropaleontology, 41: 103-108.

Didié, C., Bauch, H.A. \& Helmke, J.P. 2002. Late Quaternary deep-sea ostracodes in the polar and subpolar North Atlantic: paleoecological and paleoenvironmental implications. Palaeogeography, Palaeoclimatology, Palaeoecology, 184: 195-212.

Dingle, R.V. 1984. Mid-Cretaceous Ostracoda from Southern Africa and the Falkland Plateau. Annals of the South African Museum, 93: 97-211.

Dingle, R.V. \& Lord, A.R. 1990. Benthic ostracods and deep watermasses in the Atlantic Ocean. Palaeogeography, Palaeoclimatology, Palaeoecology, 80: 213-235.

Dingle, R.V., Lord, A.R. \& Boomer, I.D. 1990. Deep-water Quaternary Ostracoda from the continental margin off south-western Africa (SE Atlantic Ocean). Annals of the South African Museum, 99: 245-366.

Doruk, N. 1973. On Paijenborchella (Eopaijenborchella) malaiensis cymbula Ruggieri. A Stereo-Atlas of Ostracod Shells, 1: 161-164.

Ducasse, O. \& Peypouquet, J.-P. 1979. Cenozoic ostracodes: their importance for bathymetry, hydrology, and biogeography. Initial Reports of the Deep Sea Drilling Project, 48: 343-363. 
Eagar, S.H. 1965. Ostracoda of the London Clay (Ypresian) in the London Basin, 1. Reading District. Revue de Micropaléontologie, 8: 15-32.

Expedition 339 Scientists, 2013. Site U1387. In Stow, D.A.V., HernándezMolina, F.J., Alvarez Zarikian, C.A. \& the Expedition 339 Scientists, Proceedings of the Integrated Ocean Drilling Program, 339. Integrated Ocean Drilling Program Management International, Inc., Tokyo.

Faranda, C., Cipollari, P., Cosentino, D., Gliozzi, E. \& Pipponzi, G. 2008. Late Miocene ostracod assemblages from eastern Mediterranean coral reef complexes (central Crete, Greece). Revue de Micropaléontologie, 51: 287-308.

Freiwald, A. \& Mostafawi, N. 1998. Ostracods in a cold-temperate coastal environment, western Troms, northern Norway: sedimentary aspects and assemblages. Facies, 38: 255-274.

Gründel, J. 1967. Zur Grossgliederung der Ordnung Podocopida G.W. Müller, 1894 (Ostracoda). Neues Jahrbuch für Geologie und Paläontologie, Monatshefte, 6: 321-332.

Guernet, C. 2005. Ostracodes et stratigraphie du néogène et du quaternaire méditerranéens. Revue de Micropaléontologie, 48: 83-121.

Guernet, C. \& Bellier, J.-P. 2000. Ostracodes Paléocènes et Éocènes du Blake Nose (Leg ODP 171B) et évolution des environnements bathyaux au large de la Floride. Revue de Micropaléontologie, 43: 249-279.

Guernet, C. \& Fourcade, E. 1988. Cenozoic ostracodes from Hole 628A, ODP Leg 101, Bahamas. Proceedings of the Ocean Drilling Program, Scientific Results, 101: 139-151.

Hanai, T. 1957a. Study on the Ostracoda from Japan, 1. Subfamily Leptocytherinae, n. subfam. Journal of the Faculty of Science, University of Tokyo, Section 2, 10: 431-468.

Hanai, T. 1957b. Study on the Ostracoda from Japan, 3. Subfamily Cytherurinae G.W. Müller (emend. G.O. Sars 1925) and Cytheropterinae n. subfam. Journal of the Faculty of Science, University of Tokyo, Section 2, 11: 11-36.

Hazel, J.E. 1967. Classification and distribution of the recent Hemicytheridae and Trachyleberididae (Ostracoda) off northeastern North America. US Geological Survey Professional Paper, 564: 1-49.

Horne, D.J. \& Whittaker, J.E. 1985. On Eucythere declivis (Norman). A Stereo-Atlas of Ostracod Shells, 12: 1-6.

Horne, D.J. \& Whittaker, J.E. 1988. On Cytheropteron latissimum (Norman). A Stereo-Atlas of Ostracod Shells, 15: 127-132.

Hornibrook, N.B. 1952. Tertiary and Recent marine Ostracoda of New Zealand - their origin, affinities and distribution. New Zealand Geological Survey, Palaeontological Bulletin, 18: 5-82.

Hou, Y. \& Gou, Y. 2007. Fossil Ostracoda of China. Volume 2: Cytheracea and Cytherellidae. Science Publishing House, Beijing, $798 \mathrm{pp}$.

Howe, H.V. 1955. Handbook of Ostracod Taxonomy. Louisiana State University Studies, Physical Science Series, 1: 1-386.

Howe, H.V. \& graduate students, 1935. Ostracoda of the Arca zone of the Choctawhatchee Miocene of Florida. Florida Geological Survey, Geological Bulletin, 13: 1-47.

Ikeya, N. \& Suzuki, C. 1992. Distributional patterns of modern ostracodes off Shimane Peninsula, southwestern Japan Sea. Reports of the Faculty of Science, Shizuoka University, 26: 91-137.

Ishizaki, K. 1981. Ostracoda from the East China Sea. Science Reports of the Tohoku University, 2nd Series (Geology), 51: 37-65.

Ishizaki, K. \& Gunther, F. 1974. Ostracoda of the family Cytheruridae from the Gulf of Panama. Science Reports of the Tohoku University, 2nd Series (Geology), 45: 1-50.

Jones, T.R. 1849. A monograph of the Entomostraca of the Cretaceous formation of England. Monographs of the Palaeontographical Society, 3: $1-40$.

Jones, T.R. 1901. On some Carboniferous shale from Siberia. Geological Magazine (Decade 4), 8: 433-436.

Joy, J.A. \& Clark, D.L. 1977. The distribution, ecology and systematics of the benthic Ostracoda of the central Arctic Ocean. Micropaleontology, 23: $129-154$
Karanovic, I. \& Brandão, S.N. 2012. Review and phylogeny of the Recent Polycopidae (Ostracoda, Cladocopina), with descriptions of nine new species, one new genus, and one new subgenus from the deep South Atlantic. Marine Biodiversity, 42: 329-393.

Kingma, J.T. 1948. Contributions to the Knowledge of the YoungCaenozoic Ostracoda from the Malayan Region. University of Utrecht, $119 \mathrm{pp}$.

Latreille, P.A. 1802. Genera Crustaceorum et Insectorum, Tomus I, 1. Amand Koenig, Paris, 303pp.

Maddocks, R.F. 1969. Revision of recent Bairdiidae (Ostracoda). United States National Museum Bulletin, 295: 1-126.

Maddocks, R.F. 1990. Living and fossil Macrocyprididae (Ostracoda). The University of Kansas Paleontological Contributions, Monograph, 2: $1-404$.

Malz, H. 1990. Tiefseearten leben länger. Natur und Museum, 120: 139-152.

Malz, H. \& Jellinek, T. 1994. Podocopide Tiefsee-Ostracoden aus Kastengreifer-Proben im östlichen Mittelmeer ('Meteor'-Fahrt 25/leg 1: ionisches bis Levantinisches Becken). Senckenbergiana lethaea, 74: 9-32.

Mazzini, I. 2005. Taxonomy, biogeography and ecology of Quaternary benthic Ostracoda (Crustacea) from circumpolar deep water of the Emerald Basin (Southern Ocean) and the S Tasman Rise (Tasman Sea). Senckenbergiana maritima, 35: 1-119.

Moncharmont-Zei, M., Russo, B., Sgarrella, F., Bonaduce, G. \& Mascellaro, P. 1985. Paleoclimatic record from 4 cores (Gulf of Taranto, Ionian Sea). Evidence from Foraminifera and Ostracoda. Bollettino della Societá Paleontologica Italiana, 23: 21-51.

Müller, G.W. 1894. Die Ostracoden des Golfes von Neapel und der angrenzenden Meeres-Abschnitte. Fauna und Flora des Golfes von Neapel, 21: 1-404.

Müller, G.W. 1912. Ostracoda. Das Tierreich, 31: 1-434.

Neale, J.W. 1972. Kangarina septentrionalis and Paracytheridea norvegica n. spp. (Ostracoda, Cytheruridae) from offshore North Norway. Astarte, 5: 33-37.

Neale, J.W. 1973. Cluthia (Crustacea, Ostracoda), a new Pleistocene and recent Leptocytherid genus. Journal of Paleontology, 47: 683-688.

Norman, A.M. 1865. Report on the Crustacea of the deep-sea dredging off the coast of Northumberland and Durham. Transactions of the Natural History Society of Northumberland, Durham and Newcastle-upon-Tyne, 1: $12-29$.

Norman, A.M. 1867. Report on the Crustacea. In Brady, G.S. (Ed.), Reports of deep-sea dredging on the coasts of Northumberland and Durham, 1862-4. Natural History Transactions of Northumberland and Durham, 1: 12-29.

Passlow, V. \& Ayress, M.A. 1994. On Cytheropteron abyssorum Brady. A Stereo-Atlas of Ostracod Shells, 21: 63-70.

Penney, D.N. 1993. Northern North Sea benthic Ostracoda: modern distribution and palaeoenvironmental significance. Holocene, 3: 241-254.

Pirkenseer, C. \& Berger, J.P. 2011. Paleogene Ostracoda from the southern Upper Rhine Graben: Taxonomy, palaeoecology and palaeobiogeography. Palaeontographica, A, 295: 1-152.

Puri, H.S. 1954. Contribution to the study of the Miocene of the Florida Panhandle: Part 3 Ostracoda. Florida Geological Survey, Geological Bulletin, 36: 215-345.

Puri, H.S. 1957. Henryhowella, new name for Howella Puri, 1956. Journal of Paleontology, 31: 982.

Puri, H.S. \& Hulings, N.C. 1976. Designation of lectotypes of some ostracods from the Challenger Expedition. Bulletin of the British Museum (Natural History), Zoology, 29: 251-315.

Reuss, A.E. 1850. Die fossilen Entomostraceen des österreichischen Tertiärbeckens. Naturwissenschaftliche Abhandlungen, 3: 41-92.

Roemer, F.A. 1841. Die Versteinerungen des norddeutschen Kreidegebirges. Hahn'schen Hofbuchhandlung, Hannover, 145pp.

Ruan, P. \& Hao, Y. 1988. Systematic Description of Microfossils. 2. Ostracoda, Quaternary Microbiotas in the Okinawa Trough and Their Geological Significance. Geological Publishing House, Beijing, 227-395. 
Ruggieri, G. 1950. Una nuova Paijenborchella del Pliocene della Calabria. Giornale di Geologia, 2nd series, 21: 59-63.

Ruggieri, G. 1952. Gli Ostracodi delle sabbie grigie quaternarie (Milazziano) di Imola. Giornale di Geologia, 2nd series, 22: 59-115.

Ruggieri, G. 1953. Iconografia degli Ostracodi marini del Pliocene e del Pleistocene italiani. Atti della Societa Italiana di Scienze Naturali, 92: $40-56$.

Ruggieri, G. 1962. Gli Ostracodi marini del Tortoniano (Miocene medio superiore) di Enna, nella Sicilia centrale. Palaeontographia Italica, 56: 1-68.

Sars, G.O. 1866. Oversigt af Norges marine Ostracoder. Förhandlinger $i$ Videnskabs-Selskabet i Christiania, 7: 1-130 [Preprint, 1865].

Sars, G.O. 1926. Ostracoda. An Account of the Crustacea of Norway, 9, 13 and 14. Bergen Museum, 209-240.

Sars, G.O. 1928. Ostracoda. An Account of the Crustacea of Norway, 9, 15 and 16. Bergen Museum, 241-277.

Sciuto, F. 2003. Dati Preliminari sulla ostracofauna pliocenica di Capo Milazzo (Sicilia N E). Bollettino della Societá Paleontologica Italiana, 42: $179-184$.

Sissingh, W. 1972. Late Cenozoic Ostracoda of the South Agean Island Arc. Utrecht Micropaleontological Bulletins, 7: 1-187.

Stepanova, A.Y. 2006. Late Pleistocene-Holocene and Recent Ostracoda of the Laptev Sea and their importance for paleoenvironmental reconstructions. Paleontological Journal, 40: S91-S204.

Swanson, K.M. \& Ayress, M.A. 1999. Cytheropteron testudo and related species from the SW Pacific - with analyses of their soft anatomies, relationships and distribution. Senckenbergiana biologica, 79: 151-193.

Sylvester-Bradley, P.C. 1947. Some ostracod genotypes. Annals and Magazine of Natural History, Series 11, 13: 192-199.

Sylvester-Bradley, P.C. 1948. The ostracode genus Cythereis. Journal of Paleontology, 22: 792-797.

Tanaka, G., Zhou, B., Ikeya, N. \& Hasegawa, Y. 2012. Recent ostracod assemblages from Suruga Bay, Central Japan. Bulletin of Gunma Museum of Natural History, 16: 1-30.

Terquem, O. 1878. Les foraminifères et les entomostracès-ostracodes du Pliocène supérieur de l'Ile de Rhodes. Mémoires de la Société Geologique de France, Series 3, 1: 1-135.

Triebel, E. 1960. Die taxonomische Stellung und die Gattungen der Unterfamilie Macrocypridinae (Ostracoda). Senckenbergiana biologica, 41: 109-124.

van den Bold, W.A. 1946. Contribution to the Study of Ostracoda with Special Reference to the Tertiary and Cretaceous Microfauna of the Caribbean Region [Proefschrift, Rijks-Universiteit te Utrecht]. J. H. De Bussy, Amsterdam, 167 pp [reprinted in 1970 by Antiquriaat Junk, Lochem].

Ulrich, E.O. 1894. The Lower Silurian Ostracoda of Minnesota. The Geology of Minnesota (Volume 3, Part 2), 7: 629-693. Geological and Natural History Survey of Minnesota.

Ulrich, E.O. \& Bassler, R.S. 1904. Ostracoda, Miocene. The Johns Hopkins Press, Baltimore, 98-130.

Venz, K.A., Hodell, D.A., Stanton, C.S. \& Warnke, D.A. 1999. A $1.0 \mathrm{Myr}$ record of Glacial North Atlantic Intermediate Water variability from ODP site 982 in the northeast Atlantic. Paleoceanography, 14: 42-52.

Wang, P., Zhang, J., Zhao, Q. et al. 1988. Foraminifera and Ostracoda in Bottom Sediments of the East China Sea. Ocean Press, Beijing, 438pp.

Whatley, R.C. \& Ayress, M.A. 1988. Pandemic and endemic distribution patterns in Quaternary deep-sea Ostracoda. In Hanai, T., Ikeya, N. \& Ishizaki, K. (Eds), Evolutionary Biology of Ostracoda: Its Fundamentals and Applications. Kodansha, Tokyo, 739-755.

Whatley, R.C. \& Coles, G.P. 1987. The late Miocene to Quaternary Ostracoda of Leg 94, Deep Sea Drilling Project. Revista Española de Micropaleontología, 19: 33-97.
Whatley, R.C. \& Masson, D.G. 1979. The ostracod genus Cytheropteron from the Quaternary and Recent of Great Britain. Revista Española de Micropaleontología, 11: 223-277.

Whatley, R.C., Frame, P. \& Whittaker, J.E. 1978. On Rockallia enigmatica gen. et sp. nov. A Stereo-Atlas of Ostracod Shells, 5: 137-144.

Whatley, R.C., Uffenorde, H., Harlow, C.J., Downing, S. \& Kesler, K. 1982. The Rockalliidae, a new family of Cainozoic Cytheracean Ostracoda. Journal of Micropalaeontology, 1: 1-11.

Whatley, R.C., Ayress, M. \& Downing, S. 1986. Two unusual new species of the ostracod genus Cytheropteron from the late Cainozoic of the deep sea. Journal of Micropalaeontology, 5: 31-36.

Whatley, R.C., Eynon, M. \& Moguilevsky, A. 1996. Recent Ostracoda of the Scoresby Sund fjord system, East Greenland. Revista Española de Micropaleontología, 28: 5-23.

Whatley, R.C., Eynon, M. \& Moguilevsky, A. 1998. The depth distribution of Ostracoda from the Greenland Sea. Journal of Micropalaeontology, 17: $15-32$.

Witte, L. 1993. Taxonomy and biogeography of West African beach ostracods. Verhandelingen der Koninklijke Nederlandse Akademie van Wetenschappen, Afd. Natuurkunde, Eerste Reeks, 39: I-X, 1-84.

Yamaguchi, T. \& Norris, R.D. 2012. Deep-sea ostracode turnovers through the Paleocene-Eocene thermal maximum in DSDP Site 401, Bay of Biscay, North Atlantic. Marine Micropaleontology, 86-87: 32-44.

Yasuhara, M. \& Cronin, T.M. 2008. Climatic influences on deep-sea ostracode (Crustacea) diversity for the last three million years. Ecology, 89: S52-S65.

Yasuhara, M. \& Okahashi, H. in press. Quaternary deep-sea ostracode taxonomy of Ocean Drilling Program Site 980, eastern North Atlantic Ocean. Journal of Paleontology.

Yasuhara, M., Cronin, T.M., deMenocal, P.B., Okahashi, H. \& Linsley, B.K. 2008. Abrupt climate change and collapse of deep-sea ecosystems. Proceedings of the National Academy of Sciences of the United States of America, 105: 1556-1560.

Yasuhara, M., Hunt, G., Cronin, T.M. \& Okahashi, H. 2009a. Temporal latitudinal-gradient dynamics and tropical instability of deep-sea species diversity. Proceedings of the National Academy of Sciences of the United States of America, 106: 21717-21720.

Yasuhara, M., Okahashi, H. \& Cronin, T.M. 2009b. Taxonomy of Quaternary deep-sea ostracods from the western North Atlantic Ocean. Palaeontology, 52: 879-931.

Yasuhara, M., Hunt, G., van Dijken, G., Arrigo, K.R., Cronin, T.M. \& Wollenburg, J.E. 2012. Patterns and controlling factors of species diversity in the Arctic Ocean. Journal of Biogeography, 39: 2081-2088.

Yasuhara, M., Grimm, M., Brandão, S.N. et al. in press $a$. Deep sea benthic ostracodes from multiple core and epibenthic sledge samples in Icelandic waters. Polish Polar Research.

Yasuhara, M., Stepanova, A., Okahashi, H., Cronin, T.M. \& Brouwers, E.M. in press $b$. Taxonomic revision of deep-sea Ostracoda from the Arctic Ocean. Micropaleontology.

Zhao, Q.H. 2005. Late Cainozoic Ostracod faunas and paleoenvironmental changes at ODP Site 1148 South China Sea. Marine Micropaleontology, 54: $27-47$.

Zhao, Q. \& Zheng, L. 1996. Distribution of deep-sea Ostracoda in bottom sediments of the South China Sea. Acta Oceanologica Sinica, 18: 61-72, 143-146.

Zhao, Q., Whatley, R. \& Zhou, B. 2000. The taxonomy and distribution of recent species of the ostracod genus Cytheropteron in the South China Sea. Revista Española de Micropaleontología, 32: 259-281.

Zhao, Q., Wu, Y. \& Shi, X. 2011. Deep-sea Ostracoda from surface sediments of the northern Philippine Sea, northwestern Pacific. Acta Micropalaeontologica Sinica, 28: 22-34. 


\section{New publications \\ To add to your bookshelf}

The

Geological

Society

serving science \& profession

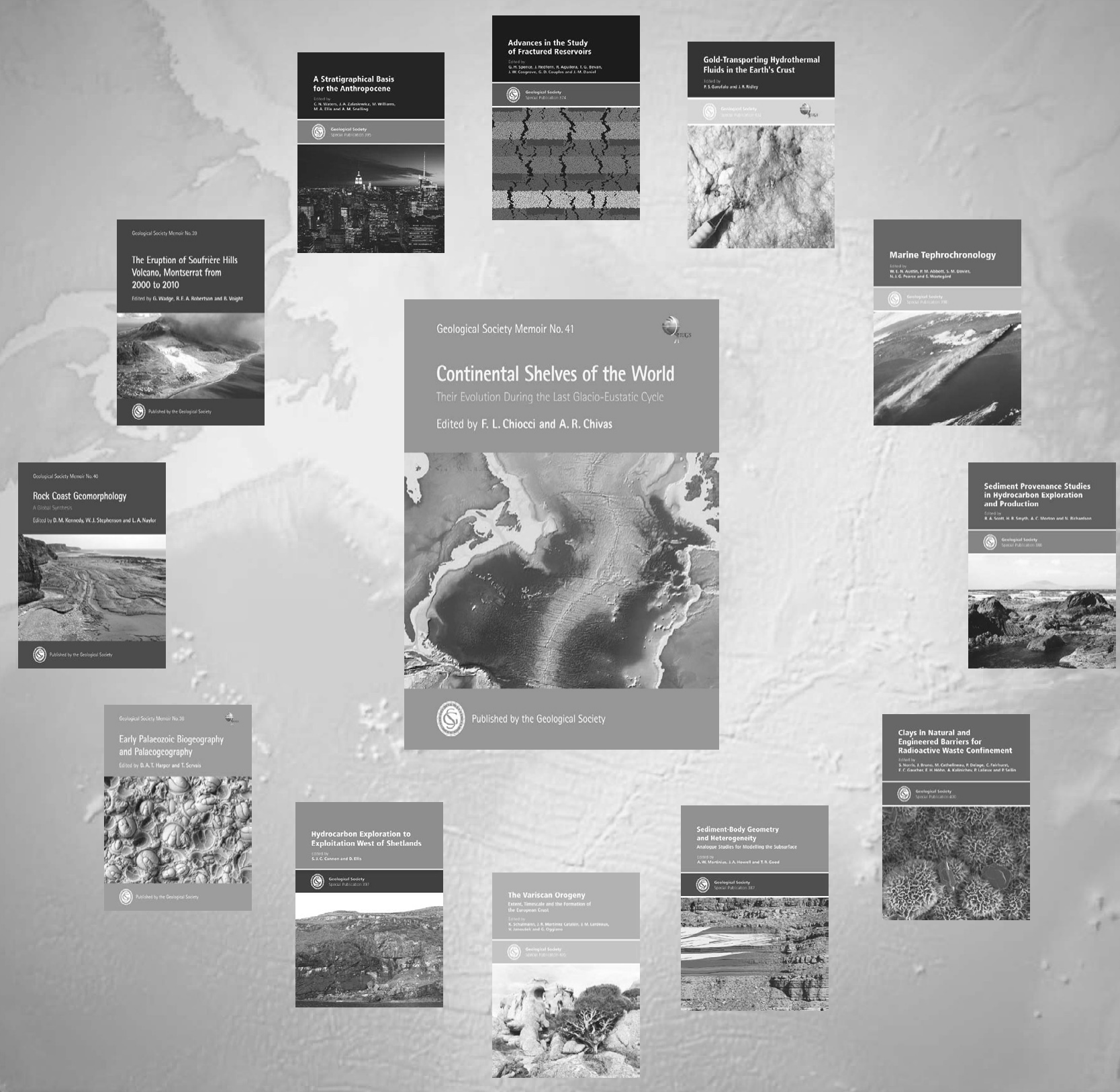

UP TO $50 \%$ DISCOUNT FOR GSL MEMBERS ON SELECTED TITLES!

Members of other societies may also qualify for discounts - contact us for details.
Browse the Online Bookshop for these and other titles from the Geological Society and other earth science publishers, visit: www.geolsoc.org.uk/bookshop 